\title{
Percepción del riesgo de inundación y anegamiento en el partido de Coronel Suárez (Argentina)
}

\author{
Perception of the risk of floods and waterlogging in Coronel Suárez's district (Argentina)
}

\author{
Historial del artículo \\ Recibido: \\ 30 de noviembre de 2020 \\ Revisado \\ 24 de mayo de 2021 \\ Aceptado: \\ 08 de junio de 2021
}

\author{
Belén Moretto ${ }^{\mathrm{a}}$, Jorge Osvaldo Gentili ${ }^{\mathrm{b}}$ \\ a Universidad Nacional del Sur, Consejo Nacional de Investigaciones Científicas y Técnicas, Argentina. Correo electrónico: \\ belen.moretto@uns.edu.ar. ORCID: https://orcid.org/0000-0003-4854-8967 \\ b Universidad Nacional del Sur, Consejo Nacional de Investigaciones Científicas y Técnicas, Argentina. Correo electrónico: \\ jogentil@uns.edu.ar. ORCID: https://orcid.org/0000-0002-4787-4667
}

\section{Palabras clave}

Inundaciones, percepción del riesgo, gestión del riesgo, cuenca del arroyo Sauce Corto.

\section{Keywords}

Floods, perception of risk, risk management, Sauce Corto basin.

\begin{abstract}
Resumen
El partido de Coronel Suárez, drenado en su mayor extensión por la cuenca del arroyo Sauce Corto, sufre el impacto de la ocurrencia de eventos de inundación y anegamiento que provocan la afectación de los modos de vida de la población y sus actividades socioeconómicas, lo que representa un riesgo para el territorio. El objetivo de este trabajo es analizar la percepción que poseen los habitantes urbanos del partido de Coronel Suárez respecto al riesgo y la gestión del riesgo de inundación y/o anegamiento. Para el presente estudio, se propone un enfoque cuanti-cualitativo mediante el método del muestreo probabilístico a partir de cuestionarios digitales. Se identificó una falta de memoria ante los impactos de estos eventos, un desconocimiento de la población de la existencia de canales de información, una débil relación entre estos y las instituciones con incumbencia en la problemática y una creencia de la sociedad de no tener la potestad o de desconocer si pueden contribuir desde su lugar a mitigar o reducir el riesgo. Asimismo, se pudo evidenciar que si bien la población da cuenta de las causas y afectaciones que producen estos eventos, mayoritariamente no perciben que exista un riesgo de inundación y/o anegamiento, hecho que puede contribuir a una cultura de prevención deficiente. Conocer y comprender la percepción del riesgo constituye un instrumento de gran relevancia para las instituciones y organismos que poseen injerencia en la temática de riesgos dado para los procesos de conocimiento, reducción y manejo del riesgo.
\end{abstract}

\begin{abstract}
Coronel Suárez's district, drained to a largest surface area by the Sauce Corto basin, suffers the impact of the occurrence of floods and waterlogging events that affect the population's ways of life and their socioeconomic activities, which results in a risk for that territory. The aim of this work is to analyze the perception of the urban resident of the Coronel Suárez's district have regarding the risk and risk management of floods and waterlogging. For that study, a quantitative-qualitative approach is proposed using the probabilistic sampling method based on digital questionnaires. A lack of memory was identified in the face of the impacts of these events, a lack of awareness by the population of the existence of information channels, a weak relationship between these and the institutions concerned in the problem, and a belief of society that they don't be able to have the power or do not know whether they can contribute from their place to mitigate or reduce risk. It was also evident that, although the population is aware of the causes and effects of these events, most of them do not perceive that there is a risk of flooding and/or waterlogging, a fact that can contribute to a deficient prevention culture. Knowing and understanding the perception of risk is a tool of great relevance for institutions and organizations that have interference in the issue of risks given for the processes of knowledge, reduction and risk management.
\end{abstract}




\section{Introducción}

La década del 90, proclamada por las Naciones Unidas como el "Decenio Internacional para la Reducción de los Desastres", dio lugar a un cambio significativo en el abordaje de la literatura del riesgo. Es así como ya no se lo considera como la ocurrencia del desastre en sí, el cual respondía a una secuencia cíclica de etapas sino en el riesgo que predispone el desastre, el cual es construido socialmente desde una visión más dinámica e integral. En la medida de que el riesgo es producto de procesos sociales particulares, es también producto directo o indirecto de los estilos o modelos de crecimiento y desarrollo impulsados en cada comunidad (Gellert-de Pinto, 2012). Ante esto, la United Nations General Assembly (UNGA) \& United Nations Office for Disaster Risk Redution (UNISDRR) (2016) plantean al riesgo como:

La posibilidad de que se produzcan muertes, lesiones o destrucción y daños en bienes en un sistema, una sociedad o una comunidad en un período de tiempo concreto, determinados de forma probabilística como una función de la amenaza, la exposición, la vulnerabilidad y la capacidad. (p. 15)

En este sentido, es relevante a la hora de analizar los desastres, continuando con la línea de la UNGA y UNISDRR (2016), examinar los contextos tanto socioeconómicos y culturales que dan lugar a condiciones de riesgo, como así también tener en cuenta que las personas no siempre comparten las mismas percepciones del riesgo y los factores subyacentes (Fatti \& Patel, 2013; García Acosta, 2005; Lara San Martin, 2012). Asimismo, hay que considerar que dentro de una misma sociedad existen diferentes vulnerabilidades y capacidades, signadas por edad, género, factores económicos, etnia, religión y ubicación física (World Meteorological Organization, 2008).

En los últimos años, comienza a tener mayor relevancia la gestión del riesgo de desastres basada en la comunidad la cual promueve la participación de la población a nivel local. En palabras de Lavell (2001):

La gestión del riesgo no es solo la reducción del riesgo, sino la comprensión que en términos sociales se requiere de la participación de los diversos estratos, sectores de interés y grupos representativos de conductas y modos de vida (incluso de ideologías y de perspectivas del mundo, la vida, la religión) para comprender como se construye un riesgo social, colectivo, con la concurrencia de los diversos sectores de una región, sociedad, comunidad o localidad concreta. (pp. -8-9)
En este contexto, el análisis de la percepción del riesgo constituye una herramienta fundamental para el estudio del riesgo y, por ende, de la resiliencia, de relevancia para una eficiente gestión integral del riesgo. La percepción del riesgo se construye socialmente y comprende la interacción entre individuos y grupos con el ambiente (Weber et al., 2000). La conciencia de los riesgos se encuentra en relación con las percepciones que se tienen de estos, para ello se necesita contar con una población que conozca y comprenda los riesgos a los cuales está expuesto (Lara San Martín, 2012). Por esto, es necesario conocer la percepción individual indagando acerca de las características socioeconómicas y culturales de las sociedades que puedan favorecer o impedir las acciones tendientes a la prevención, reducción y mitigación de las condiciones de riesgo (Cardona, 1993). La superposición de percepciones individuales es producto entonces de la imagen que un grupo social construye sobre el espacio inmediato al cual habita con las problemáticas o conflictividades que en él se desarrollan (Ferrari, 2012).

Los desbordes de los cursos de agua son fenómenos naturales asociados al normal funcionamiento de los sistemas fluviales en relación con excesos en la disponibilidad de agua. En la actualidad los eventos de origen hidrometeorológico son los de mayor recurrencia de manifestación en términos del impacto y las consecuencias que producen sobre un territorio. Son ejemplo de estos tipos de eventos las inundaciones y anegamientos. Una inundación sucede cuando los ríos reciben intensos aportes de agua, se superan los bordes de la sección transversal de los canales ocupando las márgenes (Díez-Herrero et al., 2008). Los procesos de anegamiento ocurren cuando durante eventos extremos de precipitación de escala temporal larga se anegan depresiones topográficas y terrenos de bajos gradientes de pendientes (Fuschini Mejía, 1994), proceso que Díez-Herrero et al. (2008) denominan inundaciones por endorreísmo. En particular, la percepción del riesgo de inundación es un proceso complejo que abarca características tanto cognitivas como afectivas (Martins et al., 2019) forjada por juicios intuitivos que los individuos de una sociedad hacen al respecto, utilizando para ello información limitada o incierta que varía de acuerdo al nivel de conocimiento que posean sobre el peligro en particular, en función de los niveles de vulnerabilidad, su ubicación geográfica y exposición ante la ocurrencia de estos eventos (Lara San Martin, 2012).

En las últimas décadas, a nivel internacional encontramos a diversos autores que determinan y analizan el grado de conocimiento de la población respecto al riesgo de desastres, a la percepción del riesgo y a la gestión del 
riesgo de desastres (Amérigo et al., 2019; Lara San Martín, 2012; Martins et al., 2019; Ramos Ribeiro et al., 2014; Santoro et al., 2019; Saurí et al., 2010; Serrato de la Cruz et al., 2016; Torró Segura et al., 2019). En el continente americano autores como Moreno et al. (2020), Muñoz-Duque \& Arroyave (2017), Navarro et al. (2016) y Rojas-Portocarrero et al. (2019) evalúan la actitud y comportamiento de una población ante el riesgo de inundación y la importancia de su incorporación en los procesos de gestión del riesgo. En Argentina, los trabajos de percepción del riesgo de inundación y anegamiento son escasos destacándose autores como Ferrari (2012), Murgida \& Gasparoto (2015), Carñel et al. (2016), Canevari et al. (2019), Mastrandrea, 2019 y Mastrandrea et al. (2019).

La evaluación concreta de la actitud de una población ante el riesgo y, con ello, el conocimiento de un factor básico en la valoración de su vulnerabilidad general, así como la búsqueda de las bases imprescindibles para poder dotarla de nuevas capacidades de defensa, exige un amplio trabajo de campo de índole interdisciplinar y, en particular, la aplicación de encuestas y entrevistas (Saurí et al., 2010). El partido de Coronel Suárez, drenado en su mayor extensión por la cuenca del arroyo Sauce Corto, sufre el impacto de la ocurrencia de eventos de inundación y anegamiento. Los eventos de excesos hídricos provocan la afectación de la infraestructura y las actividades socioeconómicas en torno a las localidades del municipio. Las inundaciones y/o anegamientos acaecidos en los años 2011, 2014, 2016, 2017, 2018 (Gentili, 2012; Moretto et al., 2018; Ortuño Cano et al., 2019) y recientemente en 2020 dan cuenta de la vigencia de la problemática. Dada la relevancia que en los últimos años ha adquirido la gestión del riesgo de desastres basada en la comunidad, se requieren mecanismos y estudios que den cuenta de la construcción social del riesgo en un territorio. La percepción individual y social influye en la respuesta ante la ocurrencia de un evento de exceso hídrico como así también en los mecanismos de prevención y preparación para disminuir o mitigar el riesgo subyacente (AlQahtany \& Abubakar, 2019). En el partido de Coronel Suárez, las evaluaciones asociados a este aspecto son incipientes, para evidenciar la necesidad de realizar trabajos que posibiliten comprender y analizar la percepción que poseen las comunidades del partido ante el riesgo de inundación y anegamiento como así las tendencias que se encuentran en dicho territorio. Permite hacer visible el riesgo y es un pilar fundamental en la construcción de estructuras efectivas y comportamientos para reducir la vulnerabilidad (Fatti \& Patel, 2013; Vogel et al., 2007) y desarrollar una cultura tendiente a minimizar, controlar y prevenir los mismos. En función de lo expuesto, surge una serie de interrogantes en el área de estudio: ¿los habitantes del partido de Coronel Suárez perciben las inundaciones y anegamientos como eventos peligrosos?; ¿la percepción que poseen es uniforme para todos los grupos etarios?; ¿la población local se percibe como un actor con un rol clave en los procesos de gestión del riesgo? y a raíz de ello ¿existe en el partido de Coronel Suárez una cultura de prevención? Conforme a lo expuesto, el objetivo de este trabajo es analizar la percepción que poseen los habitantes urbanos del partido de Coronel Suárez respecto al riesgo y la gestión del riesgo de inundación y/o anegamiento. En este sentido, el conocimiento e involucramiento de la sociedad obtiene una alta relevancia para elaborar una visión común que refleje las necesidades, aspiraciones y preocupaciones de las partes interesadas, en la gestión y reducción de los desastres. Comprender la percepción de los riesgos es una herramienta importante para ayudar a la planificación de acciones porque desde la evaluación y el conocimiento diario de una población es posible identificar el riesgo y evitar posibles eventos adversos, ya que los técnicos o políticos no tienen los mismos criterios para percibirlo (Medeiros Silva \& Barbosa Prestes, 2016).

\section{Materiales y métodos}

\section{Área de estudio}

El área de estudio se localiza en el Sistema de Ventania (sierras de Cura Malal, de Bravard, de la Ventana y de las Tunas) y su piedemonte, al suroeste de la provincia de Buenos Aires (Argentina) (figura 1). Está formado por un conjunto de elevaciones bien definidas con orientación noroeste-sureste que constituye el origen de ríos y arroyos que forman cuencas exorreicas y endorreicas. Por la vertiente norte de dicho sistema discurre el arroyo Sauce Corto, cuenca de alimentación exclusivamente pluvial (Gentili, 2012) que vierte sus aguas en las lagunas Encadenadas del Oeste emplazadas en el centro de la provincia. La cuenca se encuentra dentro de la faja de climas templados con estaciones térmicas y pluviométricas bien marcadas (Campo de Ferreras et al., 2004). Las precipitaciones tienen una elevada variabilidad interanual y estacional. Su distribución anual se caracteriza por un período más lluvioso que se extiende de octubre a marzo y con una estación relativamente seca de mayo a septiembre (Casado \& Campo, 2019). El sistema serrano actúa como una divisoria de aguas regional dando lugar a marcadas variaciones pluviométricas (Gentili \& Gil, 2013) y a un forzamiento orográfico de las precipitaciones del orden de los $110 \mathrm{~mm}$ (Casado et al., 2007). 


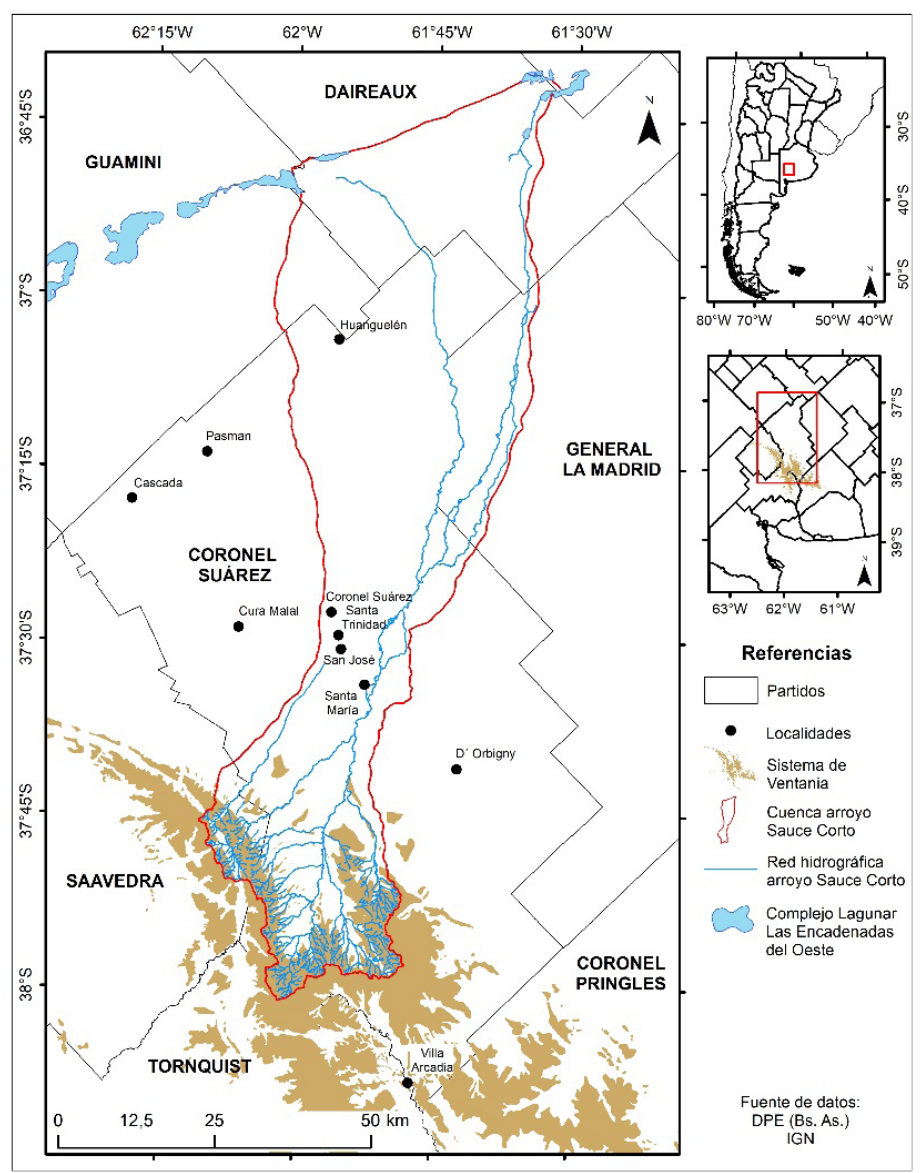

Figura 1. Área de estudio. Fuente: Elaboración propia

La cuenca del arroyo Sauce Corto posee una superficie de $9.932 \mathrm{~km}^{2}$, con una cota máxima de $975 \mathrm{msnm}$ y una cota mínima de $110 \mathrm{msnm}$, con pendientes que varían entre los $79,8^{\circ}$ y áreas totalmente planas. De manera recurrente es afectada por procesos de inundación y anegamiento que poseen un comportamiento diferencial en función de su escala temporal de manifestación y escala espacial de afectación poniendo en riesgo a la sociedad (Gentili, 2012). Ambos procesos son consecuencia de factores hidrometeorológicos, climáticos, geomorfológicos, topográficos y edafológicos que en conjunción con infraestructura vial e hidráulica en el área (puentes, terraplenes de rutas y vías férreas, alcantarillas y canales) y una sociedad expuesta y vulnerable, afectan vastos sectores de la cuenca media y baja.

La cuenca discurre a través de seis partidos de la provincia: Tornquist, Saavedra, Coronel Suárez, General La Madrid, Daireaux y Guaminí. El partido de Coronel Suárez, área del presente trabajo, es el municipio drenado en casi su totalidad por dicha cuenca (figura 1). El partido cuenta con un total de 38.320 habitantes y está conformado por las localidades de Coronel Suárez (23.621 personas) cabecera del distrito, Huanguelén (5.020 hab.), San José (2.234 hab.) -localidades consideradas como población urbana- y Santa Trinidad (1.474 hab.), Santa María (1.832 hab.), Villa Arcadia (434 hab.), Pasman (165 hab.), Cura Malal (95 hab.), D’Orbigny (20 hab.) y Cascada (11 hab.) - localidades consideradas como población rural agrupada- (INDEC, 2013) (figura 1). El partido y en especial la ciudad cabecera, como afirma (Dos Santos, 2004),

[...] no es muy diferente en sus orígenes a los conglomerados urbanos o los habitantes de los establecimientos rurales de cualquier magnitud que existen en el territorio de la provincia de Buenos Aires, salvo que lo distingue el gravitante apoyo recibido por la fundación de tres colonias con inmigrantes alemanes del Volga, en 1887. (p. 39) 


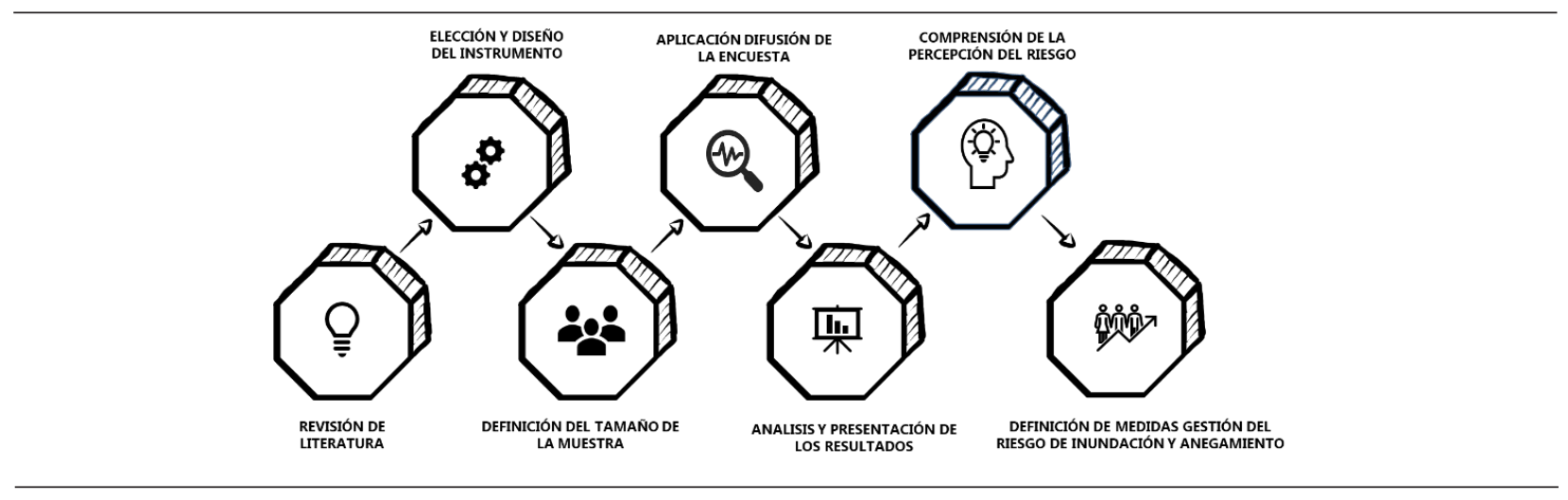

Figura 2. Diseño metodológico-técnico. Fuente: Elaboración propia con base en Hernández Sampieri et al. (2014) y United Nations (2015).

La vegetación originaria en el área es el pastizal pampeano (Cabrera, 1976). Las condiciones climáticas, topográficas, edafológicas y el contexto histórico favorecieron la expansión de la frontera agrícola, convirtiéndose en la principal fuente económica del partido signada por el desarrollo de actividades agrícolas-ganaderas. Las actividades agrícolas explican un tercio del Producto Bruto Geográfico del partido, siendo el trigo, la soja y el girasol los cultivos preponderantes. Otra actividad que cumple un rol significativo dentro del distrito es la industria manufacturera, destacándose el parque industrial Juan Zilio en las inmediaciones de la cabecera del partido ${ }^{1}$.

\section{Métodos y técnicas}

Para el desarrollo de este trabajo fue necesario evaluar tres aspectos: grado de conocimiento del riesgo, qué percepción del riesgo posee la población y cuál es el comportamiento que adopta en relación con el riesgo y gestión del riesgo de inundación y anegamiento. Si bien es difícil cuantificar la percepción que poseen las personas ante un riesgo, es posible obtener un número de encuestados con determinados comportamientos, opiniones, experiencias referidas a eventos de inundación y anegamiento. Dado el objetivo del presente estudio, se propone un enfoque mixto cuanti-cualitativo de tipo descriptivo, personal y de opinión mediante el método del muestreo probabilístico (Saurí et al., 2010; Torró Segura et al., 2019) (figura 2).

\section{Instrumento y diseño para la recolección de datos}

Se elaboró una encuesta por muestreo aleatorio simple (Hernández Sampieri et al., 2014) en la cual su formulación se basó en los criterios relativos a la naturaleza de la problemática y los factores a tener en cuenta a la hora de analizar la percepción del riesgo y la gestión del riesgo de inundación y anegamiento (Lavell, 2001; Saurí et al., 2010; United Nations General Assembly \& United Nations Office for Disaster Risk Redution, 2016). La encuesta fue estructurada en cuatro apartados para obtener una visión integral de la percepción del riesgo de inundación y anegamiento en el partido de Coronel Suárez: 1) características de la muestra, experiencia y conocimiento sobre eventos de exceso hídrico (incluye causas y afectaciones de los eventos de inundación y anegamiento); 2) canales de información, instituciones y/o organismos públicos en materia de riesgo de inundación y anegamiento; 3) acciones llevadas a cabo por instituciones u organismos públicos/privados e implicancia y 4) grado de conocimiento de la Gestión del Riesgo de Inundación y Anegamiento. Se realizó mediante la herramienta digital Google ${ }^{\circledR}$ Forms, de carácter anónimo y consta de preguntas abiertas, cerradas politómicas y de valoración (figura 2).

\section{Tamaño de la muestra}

Para determinar el tamaño de la muestra (n) (figura 2), se aplicó la siguiente ecuación (Crespo, 2013):

$$
n=\frac{Z^{2} \mathrm{NPQ}}{\mathrm{E}^{2}(\mathrm{~N}-1)+Z^{2} P Q}
$$

Donde $n$ es el tamaño de muestra; $Z$ el nivel de confianza; $N$ la población total; $E$ el error admitido y $P Q$ : varianza.

Para el estudio se definió el tamaño de la muestra con un margen de error del $8 \%$ y un nivel de confianza del

${ }^{1}$ Municipio de Coronel Suárez: https://www.coronelsuarez.gob.ar/ 


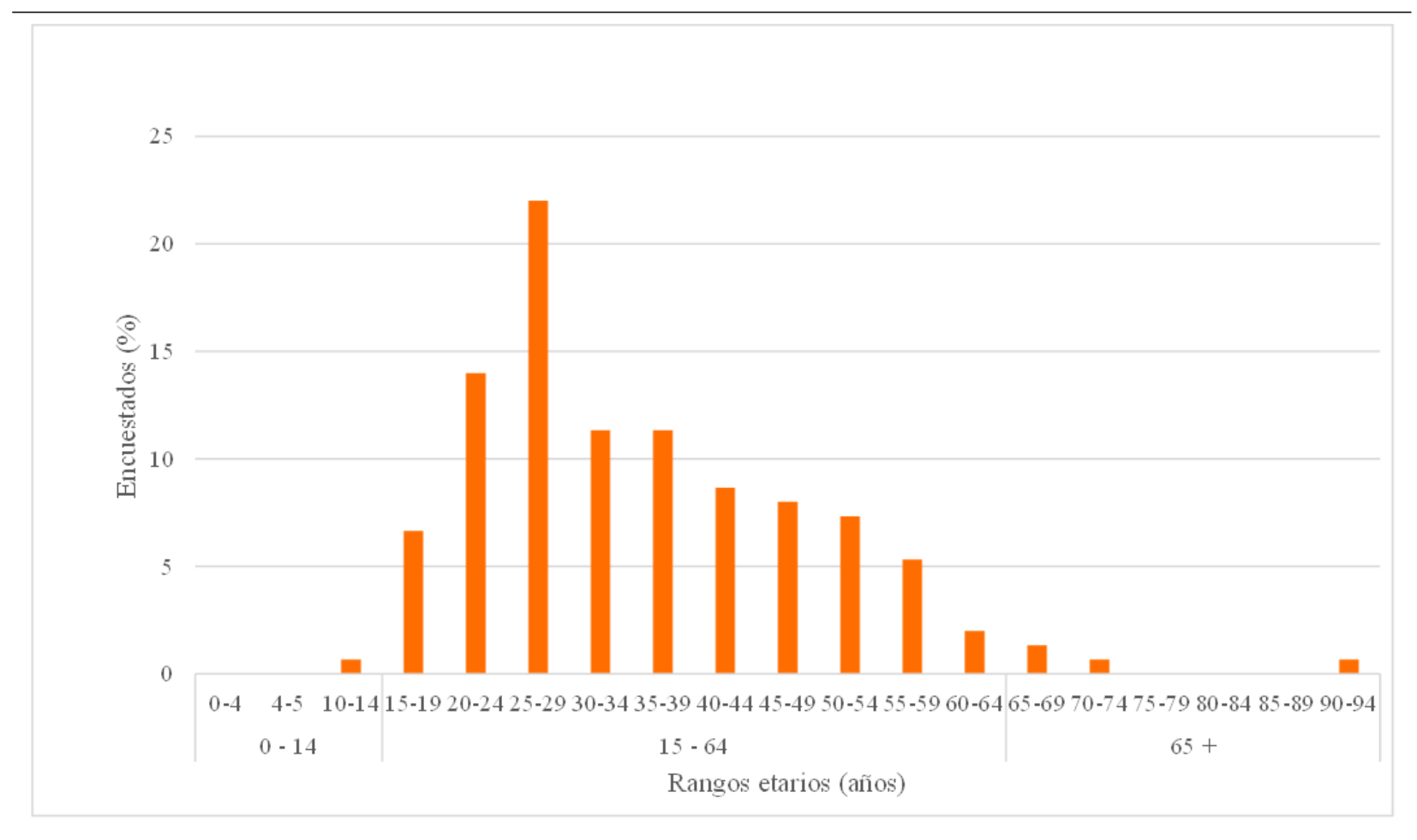

Figura 3. Proporción de la muestra en función de rangos etarios. Fuente: Elaboración propia.

$95 \%$, con una varianza del $0,5 \%$. El partido cuenta con 34.906 personas que habitan áreas urbanas y el tamaño de la muestra fue de 150 encuestas.

\section{Difusión, aplicación del instrumento y análisis de los datos}

La encuesta fue realizada en forma personal en las inmediaciones de la plaza central de la ciudad de Coronel Suárez. Asimismo, fue divulgada por diferentes medios: grupos de Facebook; correo electrónico; grupos de WhatsApp; instituciones educativas y grupo de productores agropecuarios, para abarcar a todos los sectores y habitantes de la comunidad (figura 2). Una vez recabadas las respuestas se procesaron los datos en el programa Microsoft Excel ${ }^{\circledR}$ y presentados de forma tabular o gráfica. Por último, se procedió al análisis y evaluación de las respuestas para comprender la percepción de la población con respecto al riesgo de inundación y anegamiento, lo cual resulta de gran interés para determinar el nivel de preparación de la sociedad y a partir de ello se establecieron medidas para contribuir a la gestión del riesgo (figura 2).

\section{Resultados}

Se realizaron un total de 150 encuestas sobre una población de 34.906 habitantes (INDEC, 2013). De la muestra, el 29,3
$\%$ fueron masculinos y el 70,7 \% femeninos. En cuanto a los rangos etarios, se observa que la mayor proporción de personas $(96,6 \%)$ corresponde al rango de 15 - 64 años (población económicamente activa - PEA). Dentro de dicho rango, el quinquenio de $25-29$ años ( $22 \%$ del total), seguido por el de $20-24$ (14\% del total) y los quinquenios entre $30-34$ y $35-39$ con $11,33 \%$ del total representan el mayor número de encuestados (figura 3). La mayor parte de la población encuestada pertenece a la localidad de Coronel Suárez (73 \%), principal ciudad y de mayor dinamismo del distrito, seguida por Santa María (11\%). El resto representa proporciones menores al $10 \%$ respecto al total.

\section{Eventos de excesos hídricos: experiencia, conocimiento y afectaciones}

Una de las preguntas de la encuesta indaga acerca del grado de conocimiento que la población tiene respecto de si los eventos de inundación y anegamiento corresponden al mismo proceso. Un $64 \%$ de la población coincide en que no se trata de fenómenos iguales (figura 4a). De esa cantidad, se observa que las personas que afirman conocer las diferencias son las comprendidas en los rangos etarios quinquenales de 10-14 y 55 - 59 años y la población comprendida de los 65 años y más (figura 4b). Ello puede 
a)

b)
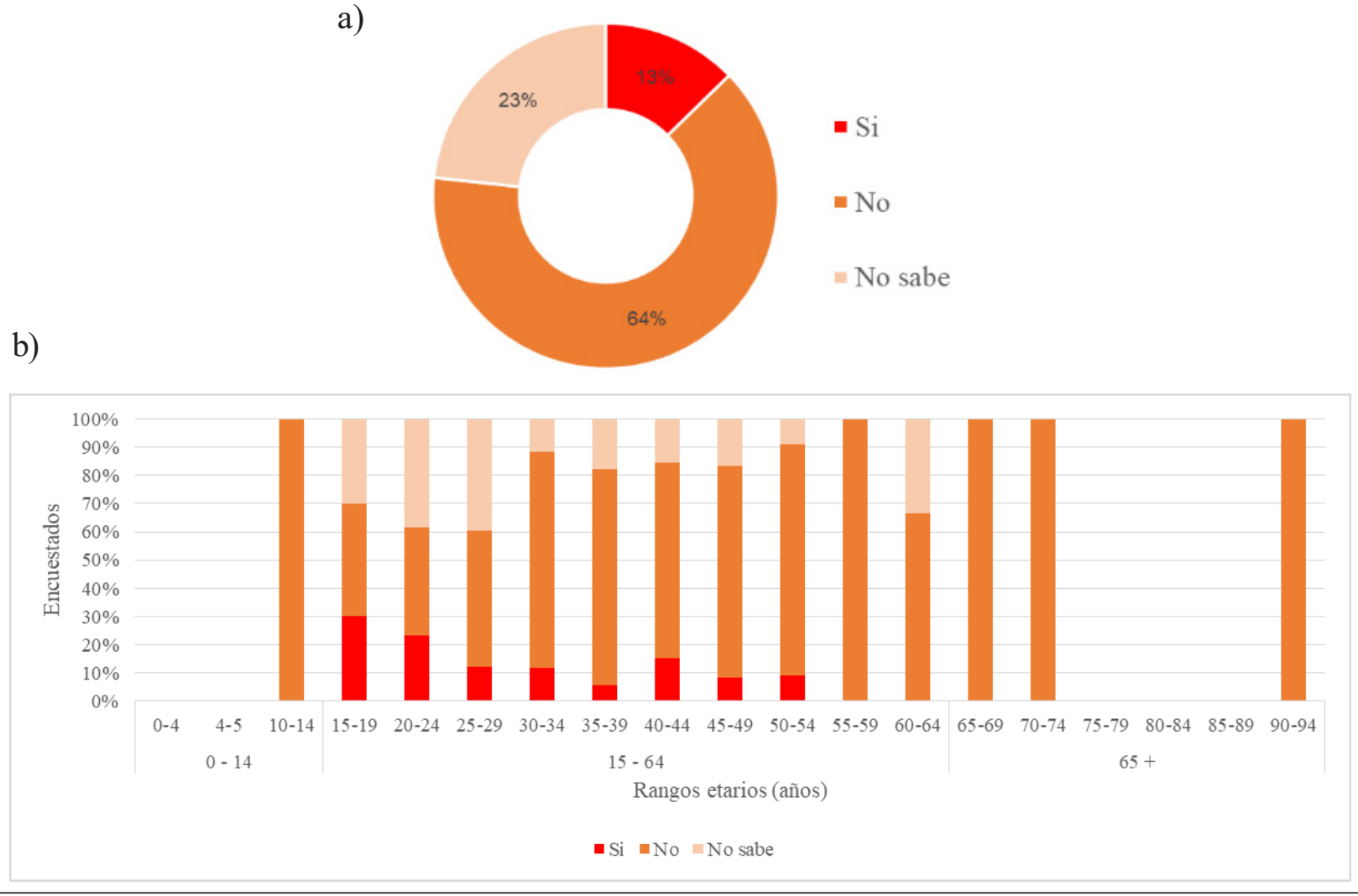

Figura 4. Proporción de población que considera o no y que no sabe si las inundaciones y anegamientos son los mismos fenómenos según: a) total de la muestra (arriba) y b) rangos etarios (abajo). Fuente: Elaboración propia.

atribuirse a múltiples causas como, en el primer caso, al grado de educación, al acceso a los canales de información y de tecnología que dan lugar a la comprensión de ambos eventos y en el segundo y tercer caso se lo puede atribuir al grado de experiencia y vivencias propias de su edad. No obstante, cabe destacar que un $23 \%$ aún desconoce y un $13 \%$ consideran que se trata de fenómenos asociados al mismo proceso (figura 4 a). Las heterogeneidades se producen en los rangos de población económicamente activa (15-64 años), destacándose las principales disparidades entre los rangos de 30 a 54 años.

En lo que respecta a la experiencia personal o recuerdos de haber vivido una inundación o anegamiento, un $40 \%$ coincide en que no se produjeron o no recuerdan eventos de excesos hídricos. El resto de la muestra (60 \%) tiene presente la ocurrencia de alguno de ellos, de los cuales un $41,11 \%$ no recuerda exactamente los años. El episodio que más reconoce la población es el del año 1985, el cual forma parte de un período húmedo que comienza en 1984 y culmina luego de 31 meses en 1987, evento de gran envergadura abordado por investigadores desde diferentes disciplinas. Asimismo, se visibiliza un claro recuerdo de algunos de los eventos acaecidos en la última década: 2014, 2016, 2018 posiblemente asociado a su cercanía temporal más que a las afectaciones que ocasionaron. Los eventos de precipitación más recientes (abril y julio de 2020) que la población menciona, dieron lugar a afectaciones principalmente en la accesibilidad y tuvieron repercusión en canales informativos de la región.

La mayor parte de encuestados $(60,7 \%)$ manifestaron que su localidad no posee riesgo de inundación y/o anegamiento y solo un $32 \%$ consideran que existe dicho riesgo. Se identificó que la población de los rangos etarios de 10-14, 60-64, 65-69 y 90-94 considera en su totalidad que no existe riesgo de inundación y anegamiento (figura 5). Los únicos grupos que señalan en mayor porcentaje que existe riesgo son los de 15-19 y 70-74 (la totalidad de los encuestados de este grupo) (figura 5). Se visibiliza que no existe una percepción única y general y la baja percepción de los fenómenos estudiados como eventos peligrosos en el partido. 


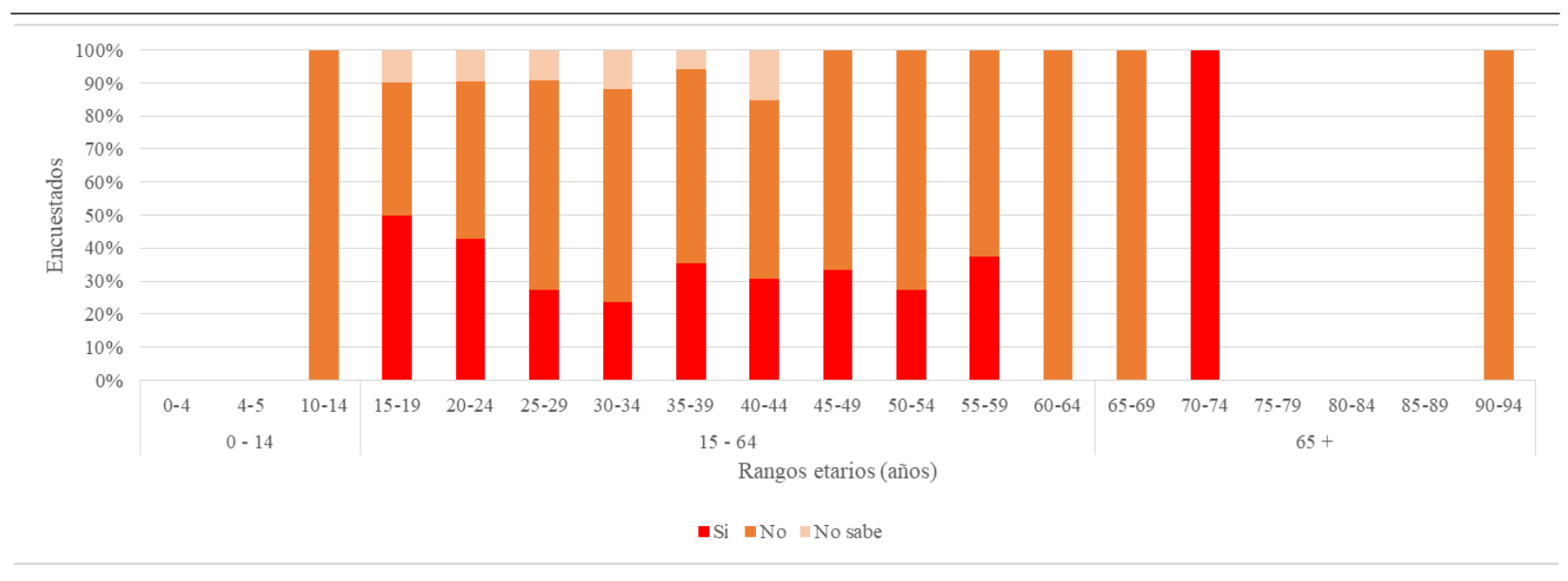

Figura 5. Proporción de encuestados que consideran si existe o no riesgo de inundación y/o anegamiento en el partido de Coronel Suárez. Fuente: Elaboración propia

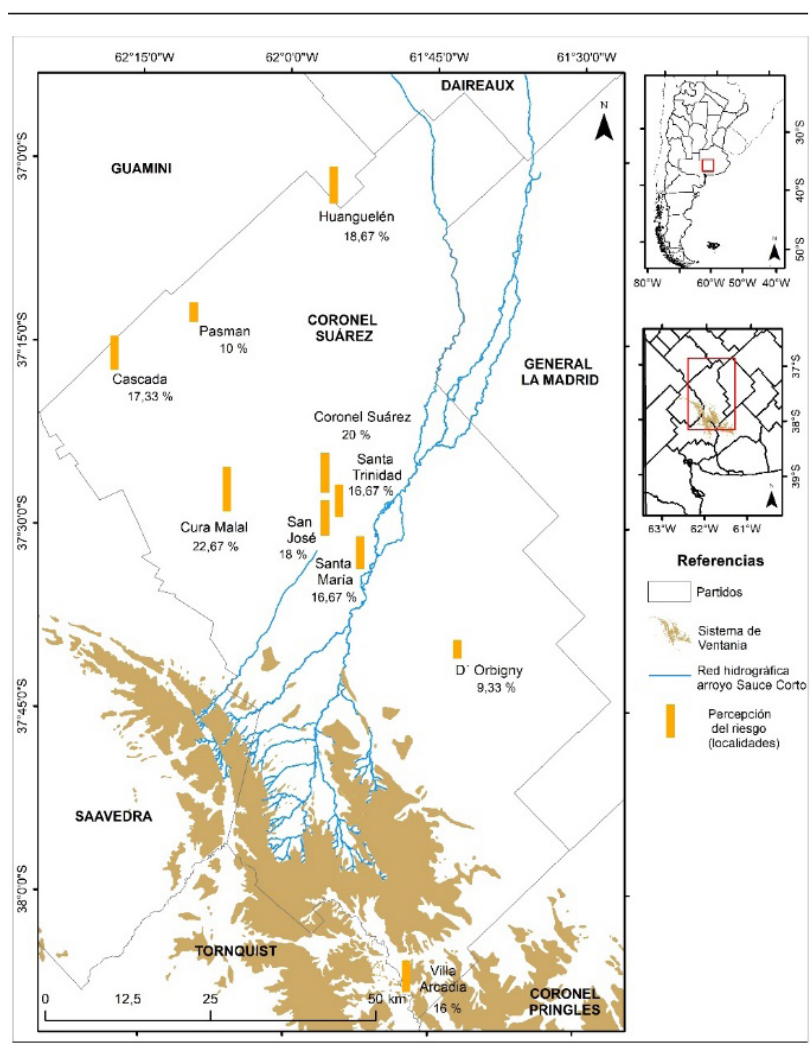

Figura 6. Proporción de encuestados que consideran que existe riesgo de inundación y/o anegamiento en las localidades del partido de Coronel Suárez. Fuente: Elaboración propia.

Otro de los puntos importantes de la encuesta es la percepción que posee la población sobre el riesgo de inundación en las restantes localidades del partido (respuesta de opción múltiple). Al respecto, un 44 $\%$ de los encuestados desconoce si el resto de las localidades del partido de Coronel Suárez presentan riesgo de inundación y/o anegamiento. La localidad percibida con mayor riesgo es Cura Malal y en segundo lugar Coronel Suárez (figura 6). Este resultado puede deberse a que ambas localidades son aquellas que se encuentran cercanas a los arroyos Cura Malal y Sauce Corto respectivamente (cursos de agua más importantes de la vertiente norte del Sistema de Ventania), por lo tanto, están más expuestas a las crecidas.

Conocer y comprender las causas que dan lugar a la ocurrencia de riesgo de inundación y anegamiento es importante para identificar los aciertos y errores y observar cuál es el grado de conocimiento de la población en relación con su entorno habitable. En una de las preguntas realizadas en el cuestionario se observó que un $71 \%$ de la población afirma que se debe a la combinación de causas naturales y sociales y en menor proporción un $12,7 \%$ con respecto a la primera de ellas. La primera afirmación vislumbra que la población conoce, en primera instancia, algunos procesos y sucesos ambientales que suceden en su partido.

En lo que concierne a la combinación de causas naturales y sociales, una gran proporción de la población considera que las causas que conllevan la ocurrencia de este tipo de eventos deviene en naturales cuando se producen eventos de precipitación intensos en poco tiempo y por la estacionalidad de las mismas; a lo que algunos agregaron la pendiente regional del terreno y en menor proporción aclararon que también se debe al mal drenaje de los suelos. Así lo demuestra una de las encuestadas que afirma que: 


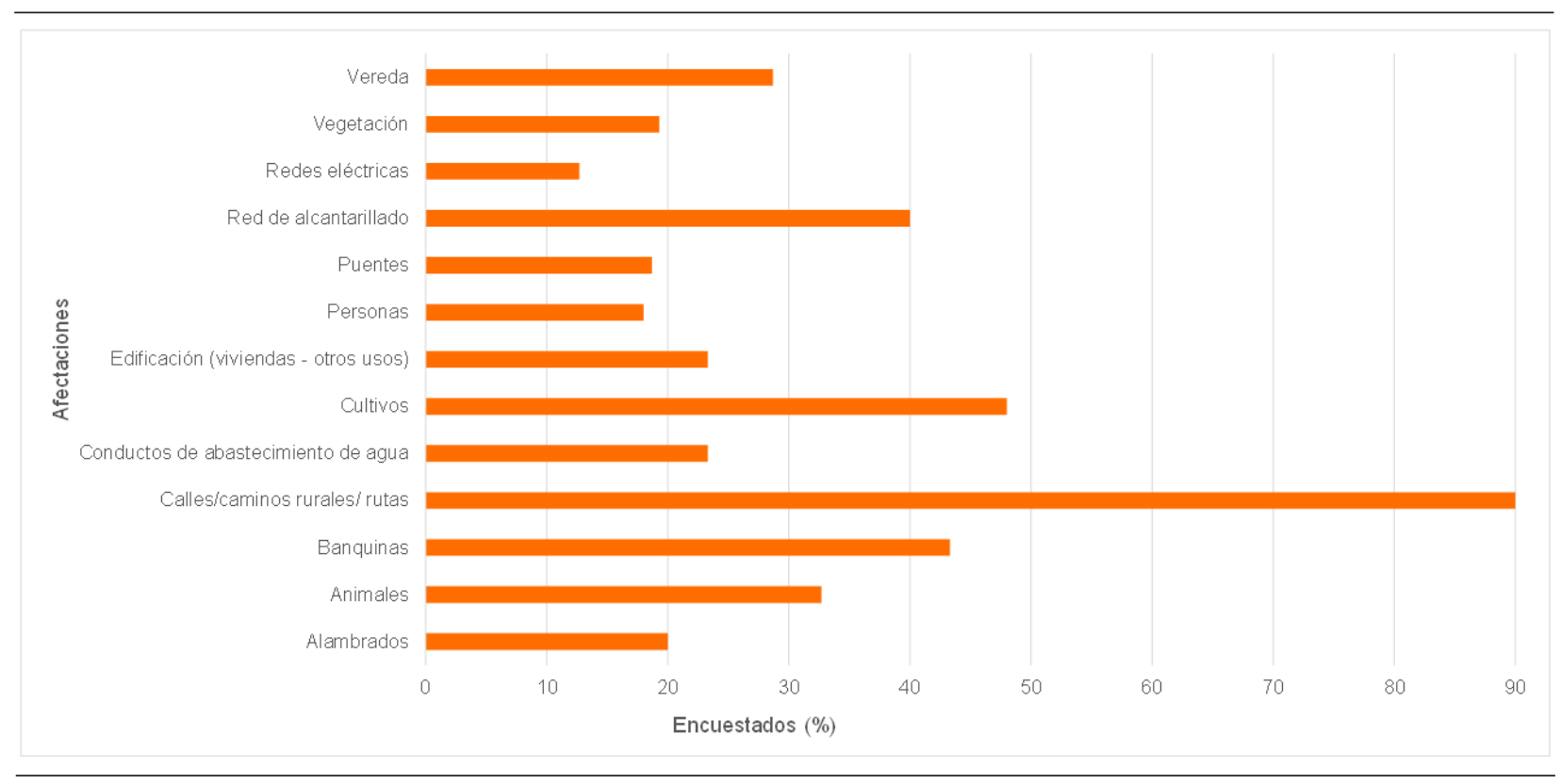

Figura 7. Principales afectaciones identificadas ante la ocurrencia de inundación y/o anegamiento. Fuente: Elaboración propia.

[...] Coronel Suárez es una zona baja en referencia a Sierra de la Ventana; y al tener un arroyo muy cercano a nuestra localidad el cual baja desde las Sierras, si se da el caso de que allá sufran una inundación o simplemente un porcentaje alto de milímetros que llueva allá, en pocas horas el agua llega a nuestra localidad y el arroyo se desborda (cómo ya ha pasado). (comunicación personal, s/f)

A ello, entre las causas producidas por la sociedad indicaron: falta de planificación y ausencia de desagües en algunos sectores, calles de tierras mal terraplenadas, pocas bocas de tormenta, falta de limpieza de canales subterráneos y presencia de canales clandestinos, poca conciencia social del desechó de residuos en lugares indebidos, calles de tierra sin entoscar, producción agrícola e irrigación excesiva, la no intervención del Estado o la mala o inconclusa infraestructura existente. En este sentido, algunas de las respuestas de los encuestados fueron:

[...] en el caso de que el caudal de agua sea muy grande, considero que no tenemos la infraestructura necesaria para no sufrir una inundación. Y por otro lado, en momentos en los cuales se registraron grandes caudales de lluvia en la zona, los campos sufrieron inundaciones, ya que tenemos las napas a muy poca profundidad, lo que hacía que el agua no drene, colapsando los campos; [...] se saca piedras en los pueblos alemanes a $15 \mathrm{~km}$ de nuestra ciudad y se inundan caminos aledaños; [...] las causas sociales son las obras como carreteras, caminos vecinales, que contienen el agua, y el escaso mantenimiento que realiza el estado municipal, en algunos casos, [...] construir donde no se debe y mal, por ejemplo las vías de tren nunca se inundan quien las diseñó sabia como. (comunicación personal, s/f)

Otro de los aspectos a tener en cuenta en la percepción que tienen los habitantes de Coronel Suárez son las afectaciones observadas ante la ocurrencia de los eventos de excesos hídricos (respuesta de opción múltiple). En la figura 7 se observa que un $90 \%$ de la población identificó a las calles, caminos rurales y rutas como los elementos más afectados. En este sentido uno de los encuestados manifiesta: “... las causas sociales son las obras como carreteras, caminos vecinales, que contienen el agua, y el escaso mantenimiento que realiza el estado municipal, en algunos casos" (comunicación personal, s/f). Otra de las afectaciones que mayor registro tuvo fue el impacto que generan estos eventos en los cultivos. En relación con ello se ve afectada en gran medida la economía del partido, ya que son las actividades agrícolas las que explican un tercio del PGB del mismo (Ministerio de Economía de la Provincia de Buenos Aires, 2014). En tercer y cuarto lugar fueron seleccionadas las banquinas relacionadas con la primera afectación y la red de alcantarillado, que según los propios habitantes se encuentran en mal estado por la falta de limpieza. Por último y en menor proporción, los elementos identificados fueron las redes eléctricas, los puentes y personas (figura 7). 


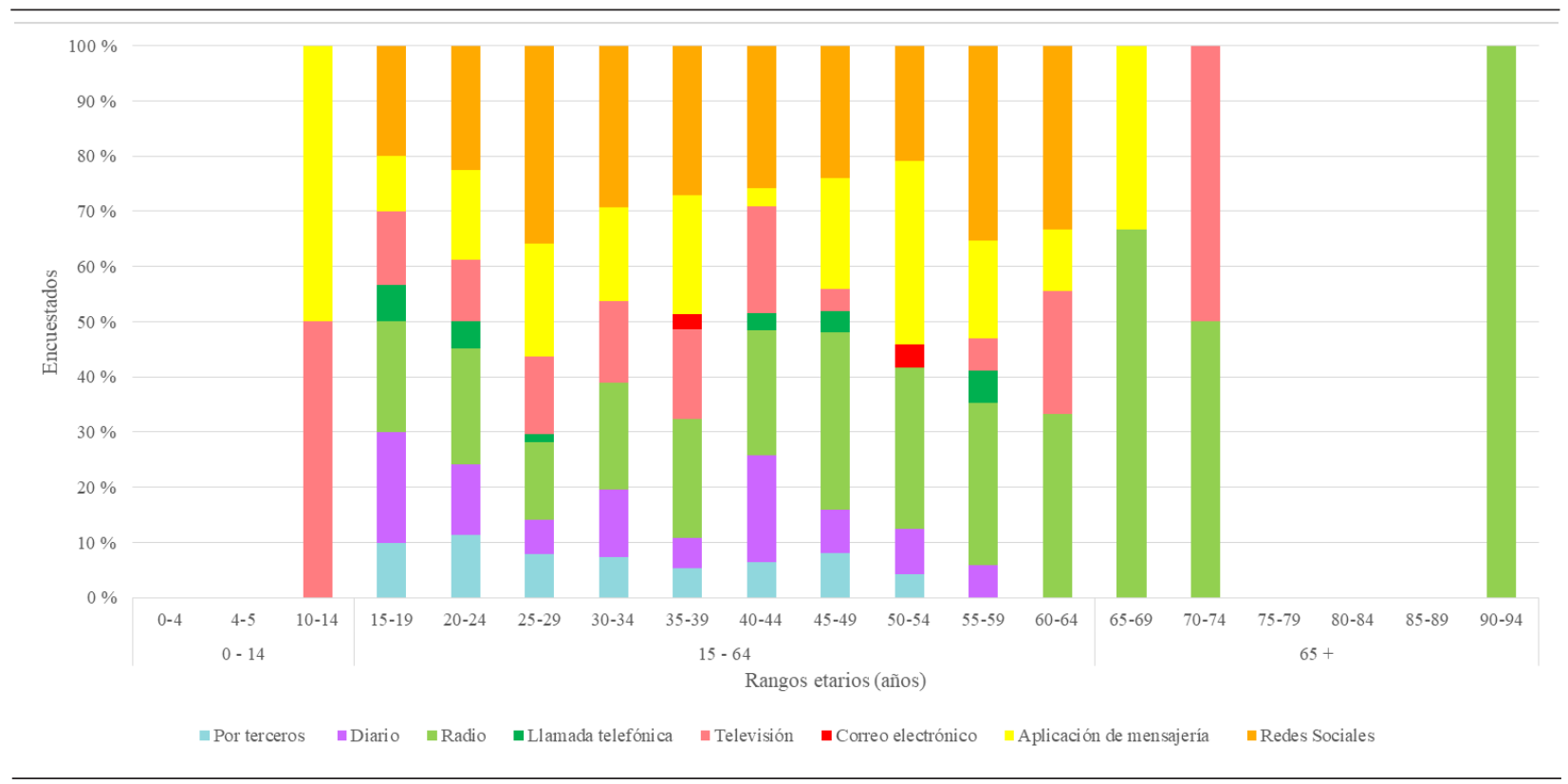

Figura 8. Medios por los cuales se anoticia primeramente ante la ocurrencia de inundación y/o anegamiento en función de los rangos etarios. Fuente: Elaboración propia..

Canales de información, instituciones y/u organismos públicos en materia de riesgo de inundación y anegamiento

El éxito de una buena gobernanza del riesgo depende de una buena comunicación de los peligros. En este sentido, en la gestión del riesgo la comunicación es una estrategia integral y planificada que la acompaña ante una emergencia o desastre. Los aspectos de comunicación pública y social y la relación con los medios de información se han convertido en una variable esencial para la gestión eficiente de la emergencia. En este sentido, en la figura 8 se observa cómo se anoticia la población acerca de la ocurrencia de un evento de inundación y/o anegamiento (respuesta de opción múltiple). Un $64 \%$ de la muestra coincide en que recibe información a través de las redes sociales (los grupos etarios entre los 15-64 años). La radio continúa siendo de gran utilidad, ya sea por su simplicidad, su alcance e inmediatez y bajo coste de acceder, en efecto, un $52 \%$ de los encuestados lo confirma. Se observa como la proporción de estas respuestas aumenta conforme la edad. En tercer lugar, las aplicaciones de mensajería $(40,7 \%)$ son también medios que permiten la difusión de información ante la ocurrencia de una emergencia (grupos de 10 a 69 años). Como casos particulares cabe destacar que los grupos de 10-14 que se anotician exclusivamente por televisión y mensajería, mientras que los dos grupos mayores (70-74 y 90-94 años) usan televisión y radio.
En la figura 9 se presentan las instituciones que son de relevancia para los habitantes del partido ante la ocurrencia de un evento de exceso hídrico. Se identifica que el cuerpo de bomberos es la institución de mayor referencia para la población ( $80 \%$ de la muestra total coincidió en la respuesta). En segunda y tercera instancia identificaron a la Comisión Vial Rural (23 \%) y Defensa Civil (22 \%) respectivamente (ambas instituciones funcionan bajo la órbita municipal). De ello resulta necesario aclarar que es muy bajo el porcentaje de población que reconoció como referente durante un evento a otras de las organizaciones $\mathrm{y} / \mathrm{o}$ instituciones presentes en el territorio.

Acciones llevadas a cabo por instituciones u organismos públicos/privados en materia de inundaciones y anegamientos

Se analizó cuál es el nivel de percepción en cuanto al grado de eficacia de las principales acciones llevadas a cabo por los diferentes organismos en las etapas del proceso de la gestión del riesgo. Si bien se comprende que el riesgo no es una conjunción cíclica de etapas, la pregunta se enfocó así a fines prácticos y de fácil interpretación para toda la población. Las medidas tomadas antes de un evento son consideradas como poco eficaces por un $36 \%$ de la población y como medianamente eficaces por un $31 \%$. Durante y después del evento, un $45 \%$ y un $41 \%$, respectivamente, afirman que las acciones son medianamente eficaces. Para las tres etapas mencionadas, 


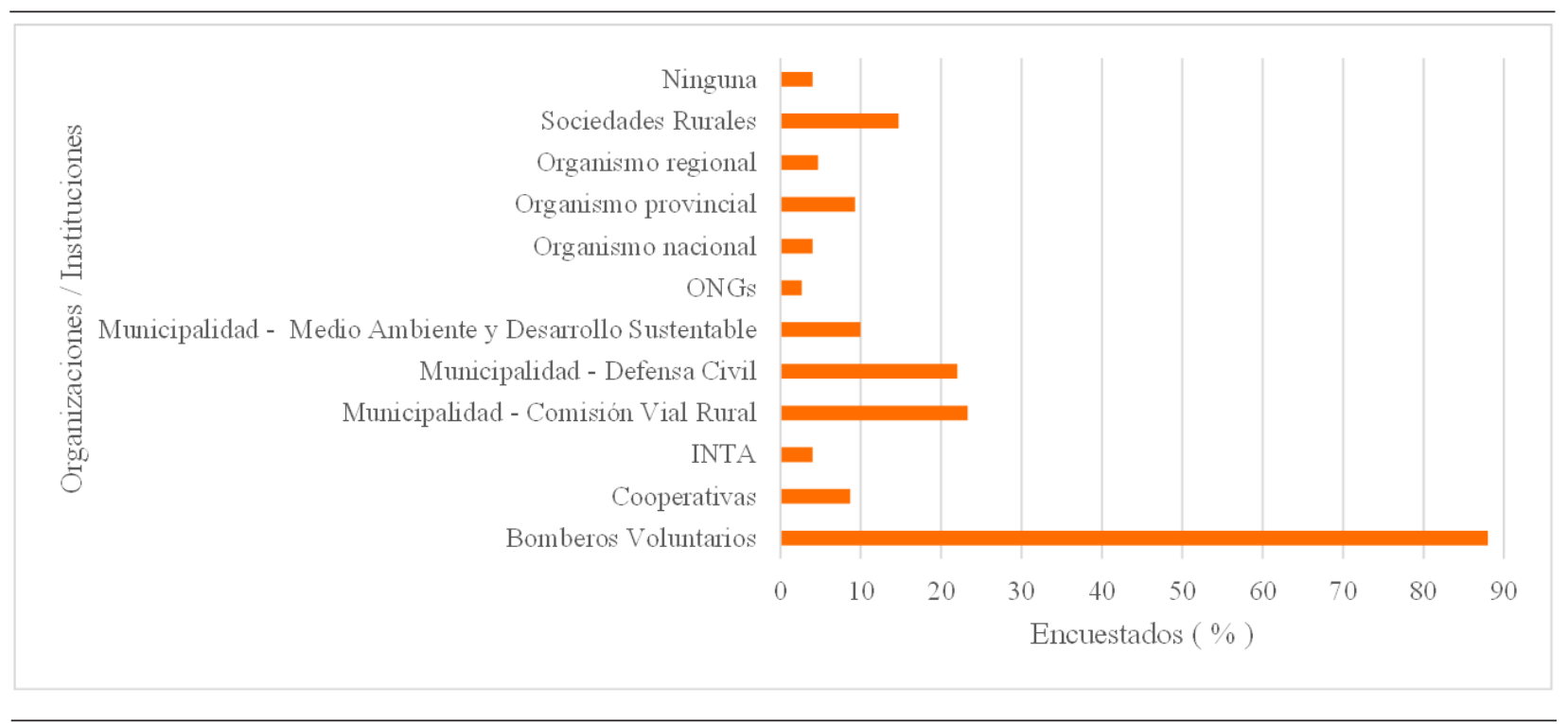

Figura 9. Instituciones, organizaciones o grupos que son de referencia al momento de la ocurrencia de inundaciones y/o anegamientos. Fuente: Elaboración propia.

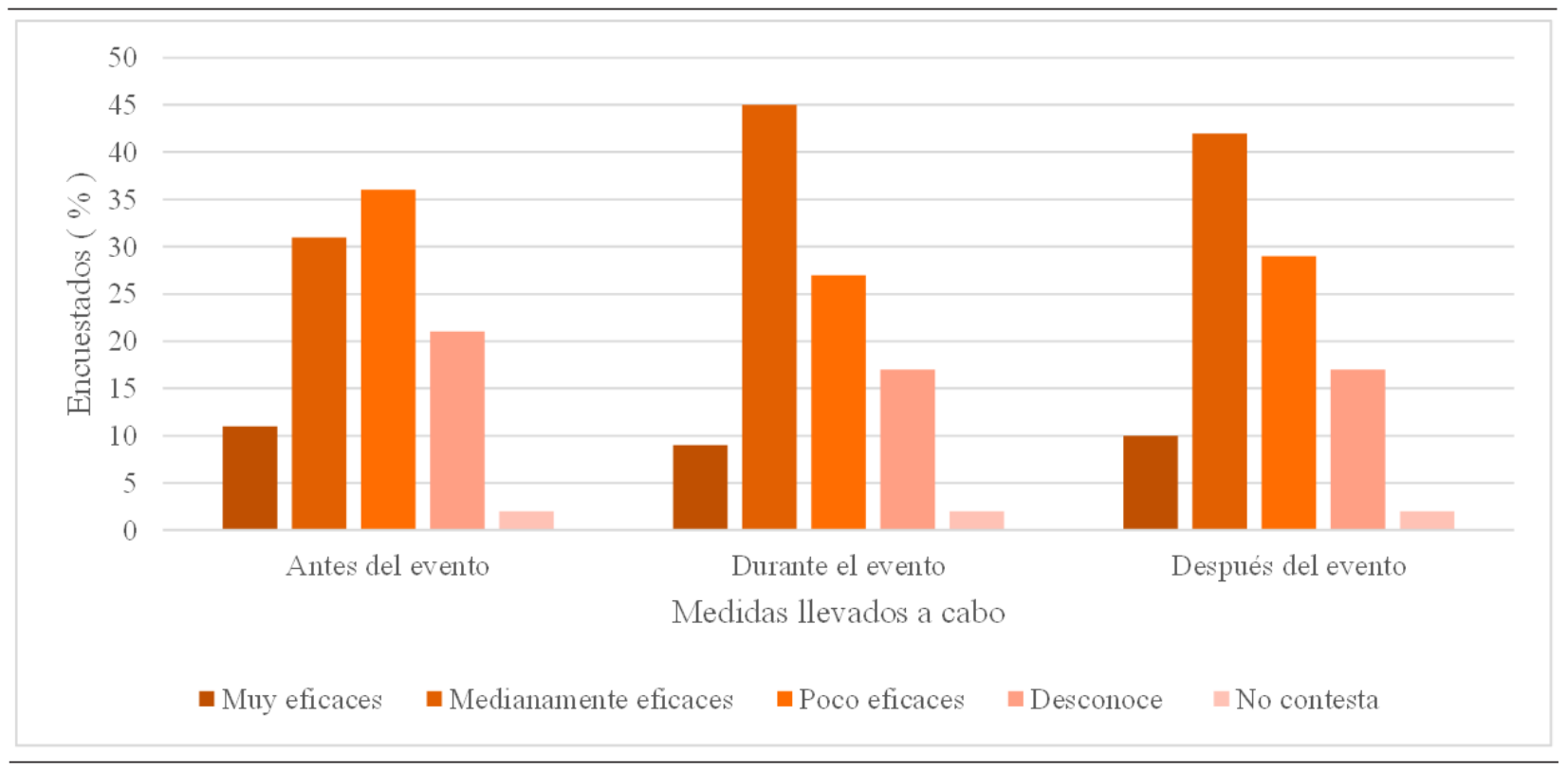

Figura 10. Grado de eficacia de las principales medidas que se realizaron a raíz de eventos de excesos hídricos. Fuente: Elaboración propia.

las medidas fueron consideradas como muy eficaces por un $10 \%$ de la población (figura 10).

Un dato para considerar es que el $80 \%$ de los encuestados no conoce canales de información. El $20 \%$ restante que sí los identificó, nombró a los Bomberos Voluntarios (coincidiendo con la valoración de esta institución con la pregunta anterior), Defensa Civil, Comisión Vial,
INTA, Sociedad Rural, la Nueva Radio Suárez 101.3 y 96.1 y por Facebook. Este resultado puede suponer la falta de difusión de la existencia de canales informativos; el déficit de interrelación y coordinación interinstitucional vertical u horizontal y la participación ciudadana en estos procesos. Se desprende de ello una débil capacidad de actuación de la población ante la ocurrencia de una inundación o anegamiento. 
Implicancia y grado de conocimiento en la gestión del riesgo de inundación y anegamiento

En relación con el grado de conocimiento de la población respecto de las implicaciones de la gestión del riesgo se observa que un $54 \%$ de la muestra desconoce tal término y un $26 \%$ no conoce su significado. Indirectamente es posible inferir la nula o escasa participación (en términos conscientes) en los procesos de gestión del riesgo de la población en el nivel local.

Otra de las preguntas del cuestionario indagaba si creían que como ciudadanos eran capaces de contribuir a la mitigación de inundaciones y/o anegamientos. Un $28 \%$ y un 36 $\%$ consideran que no tienen la potestad para contribuir y desconocen si pueden realizarlo respectivamente. Un $36 \%$ de los encuestados cree que tiene la capacidad de participar. La mayor proporción de estos últimos coincidió en que pueden involucrarse siendo responsables como ciudadanos en el cuidado del ambiente principalmente en la eliminación de residuos (en desagües, caminos, alcantarillas, canales, banquinas o cordones cuneta que impidan la circulación rápida de agua). En este sentido, una encuestada expresa "en muchos de los casos las inundaciones se dan porque las alcantarillas no pueden absorber el caudal de agua y cumplir su función ya que se encuentran desbordadas de basura" (comunicación personal, s/f). Ante esto, algunos propusieron no solo la concientización sino también la posibilidad de reciclar a partir del uso de puntos limpios ubicados en distintos sectores de la localidad. Otro de los aspectos que sobresalió es la propuesta de colaboración activa en diferentes acciones: ayudar a quienes se encontraban en situación de vulnerabilidad, ofreciendo vehículos que sean aptos ante una inundación e informarse sobre el riesgo y su gestión. Algunas de las respuestas fueron: "si tuviera información correcta o estaría informada del tema, podría comunicarlo mediante mis redes sociales"; "participando en actividades públicas relacionadas con mi región, y conociendo las problemáticas actuales"; "haciendo un grupo de cada zona que se dedique a realizar y aportar ideas, que sean loables y se lleven a cabo" (comunicaciones personales, s/f).

Existe y se observa una tendencia en el imaginario social a considerar que solo la información técnica es de relevancia o que los organismos estatales son los que se tienen que ocupar de los problemas ambientales. En relación con ello, algunos encuestados consideran que solo pueden contribuir a la gestión del riesgo si pertenecen a una institución o agrupación ya sea de productores o de injerencia a nivel local relacionados con la temática. $\mathrm{Al}$ respecto, uno de los vecinos respondió "si los estudios se hacen bien, y los trabajos a conciencia, serviría de mucho" considerando por tanto su incapacidad de ser partícipe de la reducción del riesgo.

\section{Discusión}

Para comprender la construcción social del riesgo de inundación y anegamiento en el partido de Coronel Suárez fue necesario realizar un diagnóstico de la situación que se requiere mejorar. Para ello, en primera instancia se buscó detectar las necesidades sociales presentes en determinadas condiciones de riesgo (Ulloa, 2011) mediante la realizacion de encuestas.

El partido de Coronel Suárez sufre el impacto de la ocurrencia de eventos de inundación y anegamiento y si bien estos eventos no son altamente destructivos, sí afectan constantemente a las diferentes actividades que realiza la sociedad y en particular a las zonas rurales. Si bien a grandes rasgos se visibiliza que la población reconoce la diferencia entre ambos tipos de eventos de excesos hídricos, fehacientemente no puede desprenderse que asimilen la diferencia exacta entre los mismos dada la complejidad de ambos procesos y debido a que no se indaga profundizando en lo que conllevan los mismos. De este análisis, se desprende que muchos de los encuestados no se encuentran informados o no conocen qué son eventos de origen hidrometeorológico e hidroclimático que devienen de procesos diferentes y que su impacto es diferencial en escala espacial y temporal, por tanto, es de gran relevancia que la población entienda y sea consciente de la diferencia entre ambos eventos.

En lo que respecta a la experiencia personal o recuerdos de haber vivido una inundación o anegamiento, se observa la falta de memoria de la población ante la ocurrencia de dichos eventos y en este sentido la carencia de mecanismos que pueden conducir a un afrontamiento adecuado del riesgo. El contexto socioeconómico y las experiencias personales tienen injerencia directa en la percepción que pueden tener los individuos frente al riesgo. En relación con la experiencia personal ante inundaciones, aquellos individuos que experimentan y recuerdan la ocurrencia, reconocen la existencia del riesgo, conclusiones que se encuentran en línea con lo analizado por Diakakis et al. (2018), Lara et al. (2010), Muñoz-Duque \& Arroyave (2017), Rana et al. (2020), Tasantab et al. (2020) y Wachinger et al. (2013), entre otros. Este resultado podría conjugarse como un factor predictor de relevancia asociado a un mayor nivel de percepción del riesgo. No obstante, ello, un dato de interés es que un porcentaje significativo no recuerda años específicos en los que se 
produjeron los eventos. En el área, entre 1977 y hasta la actualidad ocurrieron catorce eventos de excesos hídricos en donde si bien los impactos fueron diferenciales, irrumpió el modo de vida de la población. De todos los eventos acaecidos, el que más reconoce la población es el del año 1985 debido a su duración y efectos. En esta línea, Bradford et al. (2012) plantean que en muchas ocasiones la escasa conciencia de los individuos puede deberse a una negación ante el peligro, una falta de preocupación y una tendencia a no aceptar el riesgo que conllevan. Estas creencias representan barreras para la resiliencia de las comunidades. Por tanto, como describe Muñoz-Duque \& Arroyave (2017), quienes pueden recordar un evento de inundación suelen percibir un mayor riesgo, pues las experiencias directas son más accesibles a la propia memoria. En concordancia con Wachinger et al. (2013), si bien la experiencia directa puede tener un efecto positivo también puede tener un impacto negativo ya que los eventos de baja gravedad y periodicidad pueden producir una falsa sensación de seguridad y un juicio erróneo de la capacidad de afrontamiento. En relación con esto último y retomando el factor tiempo se visibilizan dos cuestiones importantes: por un lado, que los eventos del pasado que se aluden son los de mayor envergadura y, por otro lado, se rememoran de forma moderada aquellos episodios más actuales, como por ejemplo el evento del año 2014 que fue de gran magnitud para el área. Es un tema serio debido a que la mayor proporción de los encuestados los vivenció. En muchas ocasiones, los individuos se concentran en el problema inmediato, es decir, cuando ocurre el evento y luego de ello se olvidan de lo ocurrido. A su vez, cuando no son afectados concretamente no lo asocian como una perturbación y es por ello que es muy difícil que lo recuerden como un hecho puntual y por tanto no poseen un registro claro. Todo esto refleja la falta de memoria de la sociedad como un aspecto clave a tener en cuenta ya que puede significar una limitante para que se comprenda el riesgo de inundación y anegamiento al cual están expuestos.

La percepción del riesgo difiere de un individuo a otro. Por lo general sucede que muchas personas viven en riesgo desconociendo las condiciones de peligro a las que están expuestas o por el contrario teniendo conocimiento del peligro, convive con el riesgo, asumiéndolo como algo cotidiano y familiar (Ferrari, 2012; Saurí et al., 2010; Ulloa, 2011). La mayoría de los encuestados manifestaron que su localidad no posee riesgo de inundación y/o anegamiento. Esta baja percepción puede estar asociada a una falta de información con respecto a lo que ocurre en su partido o bien influenciada por aspectos personales, culturales y sociales más complejos. Se concuerda con
Vélez \& Vallejo (2016) que el nivel de representación del riesgo que posee la población varía conforme a ellos porque se le suele prestar mayor atención a los problemas cotidianos que enfrentan (el desempleo, la escasez de alimentos, el déficit en educación y vivienda) que a los posibles riesgos ambientales que le circundan. La importancia reside en tener en cuenta las diferentes creencias que posee la población con respecto a ello, lo que posibilita percibir cuál es la visión de su propio entorno. En concordancia con AlQahtany \& Abubakar (2019), comprender que, si bien la percepción de alto riesgo se asocia con comportamientos adecuados que conducen a una mayor resiliencia, el bajo riesgo puede dar lugar a la adopción de mecanismos inadecuados. No obstante, ello, algunos estudios (Baron \& Petersen, 2015; Diakakis et al., 2018; Wachinger et al., 2013) revelan que la relación entre la percepción del riesgo y las acciones o mecanismos adoptados son débiles y en muchos casos nulos. Por tanto, al ser compleja esta interacción, se considera que, al igual que lo planteado por AlQahtany \& Abubakar (2019), la sensibilización de los individuos sobre los peligros de inundación y anegamiento pueden influir en sus actitudes para ser más proactivos. Es por esto que la conciencia de la población es un elemento central para la gestión integral del riesgo de inundación.

A menos que los ciudadanos comprendan verdaderamente los riesgos a los que se enfrentan, la planificación de una reducción significativa del mismo puede resultar ineficaz (UNGA y UNISDRR, 2016). Para entender que los desastres "no son naturales" es importante considerar las situaciones que conllevan que se produzcan. El riesgo es una función del peligro (en este caso una inundación o anegamiento), la exposición de la población y sus bienes a dicho peligro, y del grado de vulnerabilidad al que se expone la población y sus actividades (Lavell, 2001; Natenzon \& Ríos, 2015; United Nations General Assembly \& United Nations Office for Disaster Risk Redution, 2016). En relación con ello, una gran proporción de encuestados afirman que las causas provienen de la ocurrencia de lluvias intensas. En muchas oportunidades las creencias erróneas en la población están tan arraigadas que llevan a la construcción de situaciones de riesgo. Otro de los aspectos a tener en cuenta son las afectaciones ante la ocurrencia de eventos de inundación y anegamiento. Se visibiliza a las redes viales como los elementos mayormente perjudicados. Es por ello que la ocurrencia de un evento de exceso hídrico representa un riesgo, pues se puede producir una considerable obstrucción del tránsito y asimismo se puede dañar su estructura, lo que puede dar lugar a efectos negativos a mediano y largo plazo, como así también su interrupción puede tener graves consecuencias indirectas en la sociedad. 
Otra de las afectaciones que mayor connotación obtuvo fue el impacto que generan estos eventos en los cultivos. En relación con esto, se ve afectada en gran medida la economía del partido porque son las actividades agrícolas las que explican un tercio del PGB del mismo (Ministerio de Economía de la Provincia de Buenos Aires, 2014).

Para el manejo de conceptos y la apropiación del tema es fundamental la implementación de estrategias comunicativas que generen conciencia en la población más vulnerable haciendo énfasis en la prevención y en la comprensión de las posibles consecuencias del evento (Ministerio de Salud de la Nación, 2016). Una población informada tiene mayor capacidad de reaccionar y actuar sobre los riesgos que la amenazan porque está al tanto de las manifestaciones de la emergencia y de los signos del evento. Es por esto que la población es una parte importante de la vigilancia comunitaria del evento y de la notificación de nuevos casos (Arroyo Barrantes et al., 2009). Las redes sociales son los medios por los cuales las personas se anotician sobre los eventos. Crean nuevos espacios de interacción que demandan de los organismos de gestión de riesgo desastres estrategias adicionales para comunicarse con las personas e instituciones, por tanto, transforman a todos en consumidores y productores de información (Bacigalupe et al., 2016). Al igual que afirman Houston et al. (2015), las redes sociales, en este caso, no solo permiten informar al ciudadano de la ocurrencia de un evento de inundación, sino también entregar cobertura en tiempo real, enviar y recibir pedidos de ayuda, expresar emociones y experiencias propias, como así también documentar y registrar lo sucedido. Por lo tanto, las redes sociales podrían ayudar en la construcción de resiliencia ante desastres (Dufty, 2012). Si bien, las tecnologías emergentes expanden los modos de comunicación (Bacigalupe et al., 2016), los medios tradicionales continúan siendo relevantes, no solo en los grupos etarios mayores sino también en los grupos más jóvenes. En coincidencia con Arroyo Barrantes et al. (2009), la radio es reconocida por su amplia cobertura y credibilidad y por el alcance que tiene entre la población. Es el medio de comunicación que al momento de una emergencia sirve para transmitir mensajes y recomendaciones a la población. Por su parte, las aplicaciones de mensajería pueden llegar a ser una herramienta que genere caos y mayor incertidumbre, es por ello por lo que se debe realizar un uso adecuado de los medios sociales con el fin de llevar tranquilidad. En este sentido al igual que expresan Bacigalupe et al. (2016), la información que proveen los actores sociales debe ser corroborada evitando así la circulación o filtración de datos erróneos.
Las instituciones que conforman una sociedad juegan un rol fundamental en los procesos de Gestión del Riesgo para la prevención, mitigación, preparación, respuesta, recuperación y rehabilitación. En este sentido, el Marco de Sendai expresa que para:

[...] reducir el riesgo de desastres, es necesario abordar los desafíos actuales y prepararse para los futuros centrándose en las acciones siguientes: vigilar, evaluar y comprender el riesgo de desastres y compartir dicha información y la forma en que se genera; fortalecer la gobernanza y la coordinación en materia de riesgo de desastres en las instituciones y los sectores pertinentes y la participación plena y significativa de los actores pertinentes a los niveles que corresponda. (United Nations, 2015, p. 11)

Por esto, resulta de interés también indagar acerca de la percepción de la población en relación con las instituciones que tienen injerencia en materia de agua en el partido de Coronel Suárez. Al respecto, Moretto et al. (2019) identificaron las instituciones y las organizaciones locales de carácter estatal. En este sentido, el distrito cuenta con la Comisión Vial Rural, organismo municipal cuyo objetivo es mejorar, mantener y construir las vías de comunicación terrestres y sus desagües, en el ámbito rural público. Ante lo ocurrencia de eventos de inundación y anegamiento, esta comisión tiene injerencia en las medidas que ejecuta la municipalidad para mitigar los efectos de los eventos. Asimismo, la Dirección de Ambiente y Desarrollo Sustentable de Coronel Suárez participa en el mencionado comité y confecciona informes ambientales para el OPDS, la Autoridad del Agua y otros organismos estatales. A su vez, Moretto et al. (2018) y Ortuño Cano et al. (2019) identificaron a través de la prensa escrita los actores que intervienen ante la ocurrencia de eventos de inundación y anegamiento en el partido. En los anegamientos predominan actores provinciales y nacionales, a diferencia de las inundaciones, donde aparecen principalmente los actores locales durante todo el proceso (Bomberos Voluntarios, Defensa Civil). Como demuestran estudios precedentes en el área y en otras localidades de Argentina (Carñel et al., 2016) y a nivel internacional (Rojas-Portocarrero et al., 2019), el cuerpo de bomberos es la institución con mayor porcentaje de referenciación y de relevancia para la población.

Se analizó cuál es el nivel de percepción de cada uno de los individuos encuestados, en cuanto al grado de eficacia de las principales acciones llevadas a cabo por los organismos en las etapas del proceso de la gestión del riesgo. Los resultados y lo expresado por Vergara Tenorio 
et al. (2011) lleva a reflexionar y querer indagar sobre una posible desconexión entre las instituciones u organismos públicos/privados que realizan acciones de prevención. Las creencias de las personas influyen en sus preferencias sobre las estrategias para gestionar el riesgo (Tasantab et al., 2020). Además, las preferencias y opciones locales pueden entrar en conflicto con la cultura institucional (Koehler et al., 2018). Como plantean Lara et al. (2010), Lara San Martín (2012) y Tasantab et al. (2020) hay que aunar por un enfoque de intervención de abajo hacia arriba, hacia una gestión colaborativa y más proactiva del riesgo de inundación. A raíz de esto, Wachinger et al. (2013) y Diakakis et al. (2018) plantean en que, si la sociedad desconfía de las medidas que llevan a cabo las instituciones, luego no confiarán en la información que proporcionan afectando así a la comunicación de riesgo.

En este contexto, el papel del sector de comunicación es muy importante porque permite difundir el conocimiento sobre vulnerabilidad, riesgo y medidas de prevención, así como las recomendaciones para reducir los riesgos, a través de las campañas de concientización pública y medios de comunicación masivos. A largo plazo este contribuirá a formar una cultura de prevención. Existe una creencia de que son los distintos organismos públicos los que deben actuar y prevenir. Como consecuencia de la falta de conocimiento en temas de riesgo y gestión del riesgo es que la sociedad no es consciente que es una parte fundamental del proceso. Esta condición suele dar lugar a procesos de vulnerabilidad ante la falta de concientización por parte de la población producto de no saber cómo actuar. En términos de Lavell (2001), "la gestión del riesgo es un proceso que debe ser asumido por todos los sectores de la sociedad y no como suele interpretarse, únicamente por el gobierno o el Estado como garante de la seguridad de la población" (p. 9). Si bien muchos de los encuestados se creen incapaces de contribuir ante la ocurrencia de dichos eventos, un $36 \%$ considera que tienen la potestad de participar en la mitigación de dichos riesgos. En estudios similares, para una zona de mayor extensión (Área Metropolitana de Dammam), AlQahtany y Abubakar (2019) concluyeron que un 53,8 \% de los encuestados manifiesta su capacidad de participar y para el municipio de Ontinyent (provincia de Valencia) Torró Segura et al. (2019) en la misma línea observaron que un $82 \%$ estarían dispuestos a colaborar en caso de que se realizaran actividades de esta índole.

En virtud de esto, con respecto a las medidas que los ciudadanos consideran prioritarias de implementar se observa una combinación de acciones en su mayoría no estructurales con algunas estructurales, resultado en concordancia con lo expuesto por Lara et al. (2010) y Torró Segura et al. (2019). Los eventos de inundación y anegamiento debido a las crecidas no pueden y deben evitarse ya que son procesos naturales, imprescindibles para el equilibrio y funcionamiento fluvial (Ollero Ojeda, 2020). En este sentido, la sociedad debe ser capaz de adaptarse y reducir o mitigar el riesgo que conllevan para conseguir también resiliencia. Como plantea Olleros Ojeda (2014), se debe aprender de cada evento sin falsa sensación de seguridad buscando soluciones de ordenación a mediano y largo plazo de forma permanente en pos de una cultura local del riesgo que se base en conocer, reflexionar y actuar siempre con responsabilidad.

\section{Conclusiones}

El partido de Coronel Suárez presenta riesgo de inundación y anegamiento, el cual necesita ser gestionado. La Gestión del Riesgo de Desastres es un proceso que requiere de la participación de todos los actores sociales que forman parte de un territorio. Una población incapaz de visibilizar las problemáticas que lo atraviesan afecta la capacidad local de gestionar las condiciones que construyen ese riesgo. Ante ello, es la población en primer lugar la que debe percibir y tomar conciencia del riesgo al cual está expuesta y actuar en conjunto con las instituciones y organismos privados y públicos a fin de ser resilientes ante los peligros. La percepción del riesgo es subjetiva y por tanto se deben tener en cuenta todas las opiniones para entender los comportamientos, acciones y reacciones de la sociedad ante la ocurrencia en este caso de una inundación y anegamiento.

Los resultados de las encuestas visibilizan que la población conoce en primera instancia la diferencia entre una inundación y anegamiento. No obstante, ello, no se indaga en si comprenden cada proceso, pues son eventos de origen hidrometeorológico e hidroclimático respectivamente y ambos tienen un impacto diferencial y por tanto la forma de accionar es diferente. En cuanto a la experiencia personal de haber vivido una inundación o anegamiento, un $40 \%$ de la población encuestada coincide en que no se produjeron o no recuerdan eventos de excesos hídricos. El resto de la muestra $(60 \%)$ concuerdan en que se produjeron los mismos, no obstante, de ellos se desprende que un $41 \%$ no recuerda años en los cuales se produjeron. Se evidencia la falta de memoria ante los impactos de estos eventos, rasgo que sucede en la mayoría de los sitios del mundo. Al no ser afectadas constantemente por estas problemáticas, las personas olvidan que suceden, propiciando condiciones de vulnerabilidad. 
En cuanto a las causas y afectaciones producidas por estos eventos, para el primer caso la mayoría de la población coincidió en que el riesgo de inundación y anegamiento es producto de la conjunción de causas naturales (excesos de precipitación y por consiguiente el desborde de los arroyos que drenan el partido) y causas sociales como la falta de concientización de la población en arrojar residuos en lugares indebidos, la falta de limpieza de canales, alcantarillas y boca de tormenta y por ultima por la falta de gestión. Para el segundo caso, la mayoría de la población identifica a los caminos, calles y rutas como las afectaciones que mayor impacto sufren. Este punto demuestra que la población es consciente que sus acciones (algunas de ellas incluso cotidianas) pueden tener incidencia en la ocurrencia de las inundaciones y anegamientos.

El análisis permitió dar cuenta de cuáles son las instituciones de referencia que tiene la población. Un alto porcentaje concluyó que son los Bomberos Voluntarios de gran ayuda cuando se produce un evento y en socorrer a los damnificados, a diferencia de la atribución de otras instituciones. Un dato a tener en cuenta es la falta de conocimiento de la población de la existencia de canales de información, lo que supone por un lado la posibilidad de que no existan canales de información en el partido de Coronel Suárez y por otro lado el desconocimiento de la población respecto a esto, dando lugar a condiciones de vulnerabilidad institucional.

En relación con ello, surge otro de los puntos a tener en cuenta en el análisis: la percepción que tiene la población de las medidas y acciones llevadas a cabo por las instituciones y/organismos. Las acciones antes de que ocurran los eventos son percibidas como poco eficaces y las acciones llevadas a cabo durante y después del evento son medianamente eficaces. La falta de preparación antes de que ocurran los eventos conduce a sufrir en mayor medida el impacto de las inundaciones y anegamientos y en muchas oportunidades produce la falta de capacidad de actuar de la mejor manera de acuerdo al contexto en el que cada uno se encuentra.

La creencia de la sociedad de no tener la potestad o de desconocer si pueden contribuir desde su lugar a mitigar o reducir las inundaciones y/o anegamientos da cuenta de una cultura de prevención deficiente. Los habitantes del partido de Coronel Suárez si bien dieron cuenta de las causas y afectaciones que producen los eventos de excesos hídricos en su localidad no perciben que exista un riesgo de inundación y/o anegamiento, lo cual resulta paradójico. Muchas veces sucede que la población no toma conciencia del peligro al que está expuesta y de la vulnerabilidad que poseen y consideran lejano el acontecimiento de un evento de inundación y anegamiento que afecte al desarrollo sostenible de su territorio. Son los eventos de los últimos años y sus impactos en el territorio los que advierten de la vigencia de la problemática y que por tanto es necesaria gestionarla. La falta de memoria, de una cultura de prevención y de sensibilización por parte de la población da cuenta de la magnitud del proceso de construcción del riesgo en el municipio.

La metodología utilizada brindó la oportunidad de obtener un número de encuestados con determinados comportamientos, opiniones y experiencias referidas a eventos de inundación y anegamiento. No obstante, ello, en los resultados se vislumbró percepciones diferenciales según grupos etarios. Por lo tanto, se prevé la realización de encuestas a partir de una muestra estratificada para corroborar estadísticamente (mediante pruebas de hipótesis) la relación entre la percepción y los diferentes grupos etarios que conforman la población del partido de Coronel Suárez. Los formularios de Google se constituyen como una herramienta que posibilita a la persona a contestar en el momento que le sea más apropiado y le brinda libertad de expresión al ser un cuestionario de carácter anónimo. Por esto, el enfoque utilizado y las preguntas asociadas del presente estudio son extrapolables y de fácil aplicación en otras cuencas hidrográficas y áreas del planeta.

El análisis realizado permitió proponer medidas y mecanismos para contribuir a una correcta gestión integral del riesgo. En primer lugar, es necesario realizar talleres educativos para la población de forma tal que profundicen los conceptos relacionados no solo con el riesgo hídrico sino con los factores que componen su territorio y la manera en cómo los mismos se interrelacionan. Asimismo, es importante que los talleres reflejen las preocupaciones y necesidades de la comunidad. El conocimiento y experiencia que aportan los diferentes individuos es el punto de partida para una gestión del riesgo integral y eficaz en torno a la construcción de una sociedad más resiliente. Por tanto, la posibilidad de que conozcan los marcos legales, las organizaciones, los puntos críticos dentro del partido, las áreas de mayor susceptibilidad de inundación/anegamiento y la distribución de la vulnerabilidad fortalece la capacidad de resiliencia de una población. La conformación o fortalecimiento de canales que fomenten no solo la comunicación, sino también la cooperación entre la comunidad y las instituciones con el objetivo de promover la reducción del riesgo de desastres es fundamental. 
Conocer y comprender la percepción del riesgo constituye un instrumento de gran relevancia para las instituciones y organismos que poseen injerencia en la temática de riesgos dado para los procesos de conocimiento, reducción y manejo del riesgo.

\section{Agradecimientos}

El presente trabajo se desarrolló en el marco del PGI Geografía física aplicada al estudio de la interacción sociedad-naturaleza. Problemáticas a diferentes escalas témporo-espaciales (24/G078) (SGCyT-UNS) y del PEU Inundaciones: ¿qué podemos hacer? Las tecnologías colaborativas en la gestión del riesgo (SGCyEU-UNS).

Al Dr. Ing. Eduardo de Sá Pereira (AER Coronel Suárez - EEA C. Naredo - INTA) y a las Lic. en Geografía Rocío del Milagro Ceneri Pascuale y Melina Meier por su contribución en la difusión de las encuestas.

\section{Referencias}

AlQahtany, A. M., \& Abubakar, I. R. (2019). Public perception and attitudes to disaster risks in a coastal metropolis of Saudi Arabia. International Journal of Disaster Risk Reduction, 44, 101422. https://doi.org/ https://doi.org/10.1016/j.ijdrr.2019.101422

Amérigo, M., García, J., Pérez-López, R., \& Talayero, F. (2019). Retos para afrontar el cambio climático: Análisis de la Percepción del riesgo de inundación ante riadas. Papeles Del Psicólogo - Psychologist Papers, 40(3), 197-204. https://doi.org/10.23923/ pap.psicol2019.2903

Arroyo Barrantes, S., Rodríguez, M., \& R. Pérez (Eds.). (2009). Gestión de la información y comunicación en emergencias y desastres: Guía para equipos de respuesta. Organización Panamericana de la Salud. http://bvpad.indeci.gob.pe/doc/pdf/esp/doc1581/ doc1581.htm

Bacigalupe, G., Velasco, J., Rosenberg, A., \& Berrios, P. (2016). Medios sociales en la emergencia: Evidencia y recomendaciones para la gestión de desastres. Centro de Investigación para la Gestion Integrada del Riesgo de Desastres (CIGIDEN).

Baron, N., \& Petersen, L. K. (2015). Climate change or variable weather: rethinking Danish homeowners' perceptions of floods and climate. Regional
Environmental Change, 15(6), 1145-1155. https:// doi.org/10.1007/s10113-014-0701-1

Bradford, R. A., O’Sullivan, J. J., Van Der Craats, I. M., Krywkow, J., Rotko, P., Aaltonen, J., \& Schelfaut, K. (2012). Risk perception - Issues for flood management in Europe. Natural Hazards and Earth System Science, 12(7), 2299-2309. https://doi. org/10.5194/nhess-12-2299-2012

Cabrera, M. (1976). Regiones fitogeográficas de la República Argentina. En W.Kugler (Ed.), Enciclopedia Argentina de Agricultura y Jardinería (2nd ed., pp. 1-85). ACME SACI.

Campo de Ferreras, A. M., Capelli de Steffens, A., \& Diez, P. (2004). El clima del suroeste bonaerense. Universidad Nacional del Sur, Departamento de Geografía y Turismo.

Canevari, T., Banzato, G., \& Cirio, G. (2019). Percepciones de vecinos sobre problemas sociales y ambientales en el Gran La Plata, Argentina. Hacia una agenda de Gestión Integral del Territorio. Territorios, 40, 53-82. https://doi.org/10.12804/revistas.urosario. edu.co/territorios/a.6372

Cardona, O. D. (1993). Evaluación de la amenaza, la vulnerabilidad y el riesgo. Elementos para el ordenamiento y la planeación del desarrollo. En A. Maskrey (Ed.), Los desastres no son naturales. LA RED de estudios Sociales (pp. 31-63). http:// www.planesmojana.com/documentos/estudios/19 Evaluacion\%20de\%201a\%20amenaza\%281\%29\%20 Omar\%20D.\%20Cardona.pdf

Carñel, G. E., Bonnin, R. S., \& Savoy, F. (2016). Análisis sobre la percepción frente al riesgo por inundación en dos barrios de Concepción del Uruguay, Entre Ríos, Argentina. Locale, 1(1), 97-116. https://doi. org/10.14409/rl.v1i1.6266

Casado, A., \& Campo, A. M. (2019). Extremos hidroclimáticos y recursos hídricos: estado de conocimiento en el suroeste bonaerense , Argentina. Cuadernos Geográficos, 58(1), 6-26. https://doi. org/10.30827/cuadgeo.v58i1.6751

Casado, A. L., Gil, V., \& Campo, A. M. (2007). Consecuencias de la variación de la disponibilidad hídrica en la cuenca del arroyo El Belisario, Buenos Aires, Argentina. Huellas, 11, 9-26. http:/www.biblioteca. unlpam.edu.ar/pubpdf/huellas/n11a02casado.pdf 
Crespo, R. (2013). Fórmulas y apuntes de Estadística aplicada a las ciencias sociales. 62 . www.cisolog.com

Diakakis, M., Priskos, G., \& Skordoulis, M. (2018). Public perception of flood risk in flash flood prone areas of Eastern Mediterranean: The case of Attica Region in Greece. International Journal of Disaster Risk Reduction, 28, 404-413. https://doi. org/10.1016/j.ijdrr.2018.03.018

Díez Herrero, A., Lain Huerta, L., \& Llorente Isidro, M. (Eds.). (2008). Mapas de peligrosidad por avenidas e inundaciones. Guía metodológica para su elaboración. Instituto Geológico y Minero de España.

Dos Santos, H. (2004). 120 años. 1882 - 2002 en la Historia de Coronel Suárez. Talleres Gráficos de Imprenta Feller.

Dufty, N. (2012). Using social media to build community disaster resilience. Australian Journal of Emergency Management, 27(1), 40-45. https://search.informit. org/doi/10.3316/informit.046981962746932

Fatti, C. E., \& Patel, Z. (2013). Perceptions and responses to urban flood risk: Implications for climate governance in the South. Applied Geography, 36, 1322. https://doi.org/10.1016/j.apgeog.2012.06.011

Ferrari, M. P. (2012). Análisis de vulnerabilidad y percepción social de las inundaciones en la ciudad de Trelew, Argentina. Cuadernos de Geografía - Revista Colombiana de Geografia, 21(2), 99-116. https://doi. org/10.15446/rcdg.v21n2.32216

Fuschini Mejía, M. C. (1994). El agua en las llanuras. UNESCO/ORCYT.

García Acosta, V. (2005). El riesgo como construcción social y la construcción social de riesgos. Desacatos, 19, 11-24. http://www.scielo.org.mx/scielo. php? script=sci_arttext\&pid=S1607-050X200500030 0002\&lng=es\&tlng=es

Gellert-de Pinto, G. I. (2012). Latín-A: El cambio de paradigma: de la atención de desastres a la gestión del riesgo. Boletín Cientifico Sapiens Research, 2(1), 13-17. https://www.researchgate.net/publication/277258812 El_cambio_de_paradigma_De_la_atencion_de desastres_a_la_gestion_del_riesgo_Boletin_Cientifico
Gentili, J. O, \& Gil, V. (2013). Variabilidad temporal de las precipitaciones en vertientes opuestas del Sistema de Ventania, Buenos Aires, Argentina. Revista Universitaria de Geografia, 22(2), 147-166. http:// bibliotecadigital.uns.edu.ar/scielo.php?script=sci arttext\&pid $=$ S1 1852-42652013002200003\&lng $=$ es \& $\mathrm{nrm}=\mathrm{iso}$

Gentili, J.O. (2012). Hidrografia del Arroyo Sauce Corto aplicada al estudio de las inundaciones y anegamientos [Tesis Doctoral, Departamento de Geografía y Turismo, Universidad Nacional del Sur, Argentina]. http://repositoriodigital.uns.edu.ar/ handle/123456789/494

Hernández Sampieri, R., Fernández Collado, C., \& Baptista Lucio, P. (2014). Metodología de la Investigación (6ta Ed.). McGRAW-HILL.

Houston, J. B., Hawthorne, J., Perreault, M. F., Park, E. H., Goldstein Hode, M., Halliwell, M. R., \& Griffith, S. A. (2015). Social media and disasters: A functional framework for social media use in disaster planning, response, and research. Disasters, 39(1), 1-22. https://doi.org/10.1111/ disa. 12092

INDEC. (2013). Censo Nacional de Población Hogares $y$ Viviendas 2010. Base de Datos REDATAM. Definiciones de los indicadores. http://200.51.91.245/ redarg/CENSOS/CP\%0AV2 $010 \mathrm{rad} / \mathrm{Docs} /$ indicadores.pdf

Koehler, J., Rayner, S., Katuva, J., Thomson, P., \& Hope, R. (2018). A cultural theory of drinking water risks, values and institutional change. Global Environmental Change, 50, 268-277. https://doi. org/10.1016/j.gloenvcha.2018.03.006

Lara, A., Saurí, D., Ribas, A., \& Pavón, D. (2010). Social perceptions of floods and flood management in a Mediterranean area (Costa Brava, Spain). Natural Hazards and Earth System Science, 10(10), 2081-2091. https://doi.org/10.5194/ nhess-10-2081-2010

Lara San Martín, A. (2012). Percepción Social en la Gestión del Riesgo de Inundación en un área Mediterránea (Costa Brava, España) [Tesis Doctoral, Universidad de Girona, España]. http://hdl.handle. net/10803/98249 
Lavell, A. (2001). Sobre la gestión del riesgo: apuntes hacia una definición. Scripta Nova-Revista. http://cidbimena.desastres.hn/pdf/spa/doc15036/ doc15036-contenido.pdf

Martins, B., Nunes, A., Lourenço, L., \& Velez-Castro, F. (2019). Flash flood risk perception by the population of Mindelo, S. Vicente (Cape Verde). Water, 11(9), 1-14. https://doi.org/10.3390/w11091895

Mastrandrea, A. (2019). Percepción social del riesgo hídrico: análisis de los significados atribuidos a los eventos de anegamientos en el barrio Villa Talleres, ciudad de Bahía Blanca (provincia de Buenos Aires, Argentina). Estudios Socioterritoriales, 26. https://doi.org/https://doi.org/10.37838/unicen/ est.26-027

Mastrandrea, Aldana, Angeles, G., \& Olavarría, J. (2019). Evaluación de la percepción social del espacio fluvial urbanizado del arroyo Napostá Grande, Bahía Blanca, Argentina. Estudios Geográficos, 80(287), e017. https://doi.org/10.3989/estgeogr.201933.014

Medeiros Silva, M. C., \& Barbosa Prestes, M. (2016). Percepção de risco dos atores sociais em áreas metropolitanas: estudo de caso na comunidade São José - Joao Pessoa. Brasil. Revista Espacios, 37(23). https://www.revistaespacios.com/ a16v37n23/16372305.html

Ministerio de Economía de la Provincia de Buenos Aires. (2014). Programacion del Desarrollo Territorial. Diagnóstico preliminar y líneas de acción para la discusión. Tomo III. https://www.simco.rafam.ec.gba. gov.ar/inicio/ver_pdf_publicaciones/5/75

Ministerio de Salud de la Nación. (2016). Salud, comunicación y desastres. Guía básica para la comunicación de riesgo en Argentina. http://www.msal.gob.ar/images/stories/ryc/ graficos/0000000832cnt-2016-05_guia-saludcomunicacion-desastres-isbn.pdf

Moreno, M., Álvarez, H., \& Chacón, A. (2020). Estudio de percepción social en las vivencias postdesastre, para determinar el nivel de resiliencia y afectación comunitaria en el distrito de $\mathrm{La}$ Chorrera, provincia de Panamá Oeste. Revista de I+D Tecnológico, 16(1), 90-103. https://doi. org/10.33412/idt.v16.1.2432
Moretto, B., Gentili, J. O., Ortuño Cano, M., \& Campo, A. M. (2019). El agua: recurso y peligro. Análisis normativo-institucional para la vertiente norte del Sistema de Ventania (Argentina). Geográfica Digital, 16(31), 29-45. https://doi.org/https://dx.doi. org/10.30972/geo.16313598

Moretto, B., Ortuño Cano, M., Gentili, J. O., \& Campo, A. M. (2018). Eventos de inundación en la vertiente norte del sistema de Ventania: análisis a través de la prensa escrita. XII Jornadas Nacionales de Geografía Física, 64-69, Trelew, Argentina.

Muñoz-Duque, L. A., \& Arroyave, O. (2017). Percepción del riesgo y apego al lugar en población expuesta a inundación: un estudio comparativo. Pensamiento Psicológico, 15(2), 79-92. https://doi.org/10.11144/ javerianacali.ppsi15-2.pral

Murgida, A. M., \& Gasparoto, M. (2015). Percepción del riesgo y sistemas participativos de alerta temprano en Iruya, Provincia de Salta. En C. Natenzon \& D. Ríos (Eds.), Riesgos, catástrofes y vulnerabilidades. Aportes desde la Geografía y otras Ciencias Sociales para casos argentinos (pp. 75-95). Imago Mundi.

Natenzon, C., \& Ríos, D. (2015). Riesgos, catástrofes y vulnerabilidades: aportes desde la geografia y otras ciencias sociales para casos argentinos. Imago Mundi.

Navarro, O., Chaves-Castaño, L., Noreña Betancur, M. I., \& Piñeres-Sus, J. D. (2016). Percepción del riesgo y estrategias de afrontamiento en población expuesta y no expuesta al riesgo de inundación. Interamerican Journal of Psychology, 50(3), 331-346. http://www. redalyc.org/articulo.oa? $\mathrm{id}=28450492003$

Ollero Ojeda, A. (2014). Guía metodológica sobre buenas prácticas en gestión de inundaciones (Manual para gestores). http://contratoderiomatarranya.org/ documentos/Guia_BB_Gestion_inundaciones.pdf

Ollero Ojeda, A. (2020). Crecidas, inundaciones y resiliencia: restauración fluvial contra los falso mitos. En M. I. López Ortiz \& J. Melgarejo (Eds.), Riesgo de inundación en España: análisis y soluciones para la generación de territorios resilientes (pp. 549-567). Universitat d'Alacant. https://dialnet.unirioja.es/ servlet/articulo? codigo $=7587786$ 
Ortuño Cano, M., Gentili, J., Moretto, B., \& Campo, A. (2019). Eventos de exceso hídrico en la prensa escrita (Sistema de Ventania, Argentina). Boletín Geográfico, 1(41), 53-75. http://revele.uncoma.edu.ar/htdoc/ revele/index.php/geografia/article/view/2366/59029

Ramos Ribeiro, R. R., Olcina Cantos, J., \& Molina Palacios, S. (2014). Análisis de la percepción de los riesgos naturales en la Universidad de Alicante. Investigaciones Geográficas, 61, 147-157. https:// doi.org/10.14198/ingeo2014.61.10

Rana, I. A., Jamshed, A., Younas, Z. I., \& Bhatti, S. S. (2020). Characterizing flood risk perception in urban communities of Pakistan. International Journal of Disaster Risk Reduction, 46. https://doi.org/10.1016/j. ijdrr.2020.101624

Rojas-Portocarrero, W. K., Hidalgo-Egocheaga, B., Moya-Durand, C. A., Castro-Pérez, F., \& BarbozaPalomino, M. (2019). Percepción de riesgo ante las inundaciones en personas que habitan en zonas vulnerables de Lima, Perú. Revista Cubana de Salud Pública, 45(2), 1-15. https://scielosp.org/pdf/ rcsp/2019.v45n2/e1190/es

Santoro, S., Pluchinotta, I., Pagano, A., Pengal, P., Cokan, B., \& Giordano, R. (2019). Assessing stakeholders' risk perception to promote Nature Based Solutions as flood protection strategies: The case of the Glinščica river (Slovenia). Science of the Total Environment, 655, 188-201. https://doi. org/10.1016/j.scitotenv.2018.11.116

Saurí, D., Ribas, A., Lara, A., \& Pavón, D. (2010). La percepción de inundaciones: experiencias de aprendizaje en la Costa Brava. Papeles de Geografía, 51-52, 269-278. https://revistas.um.es/geografia/ article/view/114571/108571

Serrato de la Cruz, B. A., Garcia Gastelum, A., Figueroa Beltran, C., \& Pantle Cebada, D. (2016). Percepción del riesgo de inundación por desbordamiento de presa en zona urbana vulnerable. Papeles de Geografia, 62, 77-89. https://doi.org/10.6018/geografia/2016/234741

Tasantab, J. C., Gajendran, T., von Meding, J., \& Maund, K. (2020). Perceptions and deeply held beliefs about responsibility for flood risk adaptation in Accra Ghana. International Journal of Disaster Resilience in the Built Environment, 11(5), 631-644. https://doi. org/10.1108/IJDRBE-11-2019-0076
Torró Segura, M., Camarasa Belmonte, A., \& Pitarch Garrido, M. D. (2019). Percepción del riesgo de inundación en el municipio de Ontinyent (Comunitat Valenciana). Cuadernos de Geografía de La Universitat de València, 103, 117-140. https://doi. org/10.7203/cguv.103.16227

Ulloa, F. (2011). Manual de Gestión de Riesgos de Desastre para comunicadores sociales. Una guía práctica para el comunicador social comprometido en informar y formar para salvar vidas. http://repo. floodalliance.net/jspui/handle/44111/2151

United Nations. (2015). Marco de Sendai para la Reducción del Riesgo de Desastres 2015-2030. https://www.unisdr. org/files/43291_spanishsendaiframeworkfordisasterri. pdf

United Nations General Assembly (UNGA) \& United Nations Office for Disaster Risk Redution (UNISDRR). (2016). Report of the open-ended intergovernmental expert working group on indicators and terminology relating to disaster risk reduction. https://www.preventionweb.net/ files/50683_oiewgreportenglish.pdf

Vélez, J. A., \& Vallejo, A. (2016). Percepción del Riesgo en los procesos de urbanización del territorio. Entorno Geográfico, 1. https://doi.org/10.25100/eg.v0i1.7577

Vergara Tenorio, M. del C., Ellis, E. A., Alarcón, L. del C., \& Galván del Moral, U. (2011). La conceptualización de las inundaciones y la percepción del riesgo ambiental. Política y Cultura, 36, 45-69. http://www.scielo.org.mx/scielo.php?script=sci arttext\&pid=S0188-77422011000200003\&lng=es\&t $\operatorname{lng}=\mathrm{es}$

Vogel, C., Moser, S. C., Kasperson, R. E., \& Dabelko, G. D. (2007). Linking vulnerability, adaptation, and resilience science to practice: pathways, players, and partnerships. Global Environmental Change, 17(3-4), 349-364. https://doi.org/10.1016/j. gloenvcha.2007.05.002

Wachinger, G., Renn, O., Begg, C., \& Kuhlicke, C. (2013). The risk perception paradox-implications for governance and communication of natural hazards. Risk Analysis, 33(6), 1049-1065. https://doi. org/10.1111/j.1539-6924.2012.01942.x 
Weber, J., Hair, J., \& Fowler, C. (2000). Developing a measure of perceived environmental risk. The Journal of Environmental Education, 32(1), 28-35. https://doi.org/10.1080/00958960009598669

World Meteorological Organization (WMO). (2008). Organizing Community Participation for Flood Management. A Tool for Integrated Flood Management. http://www.apfm.info/pdf/ifm_tools/ Tools_Organizing_Community_Participation_for_ FM.pdf 